\title{
AMPK Preferentially Depresses Retrograde Transport of Axonal Mitochondria during Localized Nutrient Deprivation
}

\author{
Orla Watters, ${ }^{1,3}{ }^{\circledR}$ Niamh M. C. Connolly, ${ }^{1,2}{ }^{\circledR}$ Hans-Georg König, ${ }^{1}$ Heiko Düssmann, ${ }^{1,2}$ and \\ (ㄱ) Jochen H. M. Prehn ${ }^{1,2,3}$ \\ ${ }^{1}$ Centre for the Study of Neurological Disorders, ${ }^{2}$ Centre for Systems Medicine, Department of Physiology and Medical Physics, Royal College of \\ Surgeons in Ireland, Dublin 2, Ireland, and ${ }^{3}$ SFI FUTURE-NEURO Research Centre, Royal College of Surgeons in Ireland, Dublin 2, Ireland
}

Mitochondrial clusters are found at regions of high-energy demand, allowing cells to meet local metabolic requirements while maintaining neuronal homeostasis. AMP-activated protein kinase (AMPK), a key energy stress sensor, responds to increases in AMP/ATP ratio by activating multiple signaling cascades to overcome the energetic deficiency. In many neurologic conditions, the distal axon experiences energetic stress independent of the soma. Here, we used microfluidic devices to physically isolate these two neuronal structures and to investigate whether localized AMPK signaling influenced axonal mitochondrial transport. Nucleofection of primary cortical neurons, derived from E16-18 mouse embryos (both sexes), with mito-GFP allowed monitoring of the transport dynamics of mitochondria within the axon, by confocal microscopy. Pharmacological activation of AMPK at the distal axon (0.1 mM 5-aminoimidazole-4-carboxamide riboside) induced a depression of the mean frequency, velocity, and distance of retrograde mitochondrial transport in the adjacent axon. Anterograde mitochondrial transport was less sensitive to local AMPK stimulus, with the imbalance of bidirectional mitochondrial transport resulting in accumulation of mitochondria at the region of energetic stress signal. Mitochondria in the axon-rich white matter of the brain rely heavily on lactate as a substrate for ATP synthesis. Interestingly, localized inhibition of lactate uptake (10 nM ARC155858) reduced mitochondrial transport in the adjacent axon in all parameters measured, similar to that observed by 5aminoimidazole-4-carboxamide riboside treatment. Coaddition of compound $\mathrm{C}$ restored all parameters measured to baseline levels, confirming the involvement of AMPK. This study highlights a role of AMPK signaling in the depression of axonal mitochondrial mobility during localized energetic stress.

Key words: mitochondrial transport; metabolic stress; axonal injury; AMPK; lactate; microfluidic device

Significance Statement

As the main providers of cellular energy, the dynamic transport of mitochondria within the neuron allows for clustering at regions of high-energy demand. Here we investigate whether acute changes in energetic stress signal in the spatially isolated axon would alter mitochondrial transport in this local region. Both direct and indirect activation of AMP-activated protein kinase isolated to the distal axon induced a rapid, marked depression in local mitochondrial transport. This work highlights the ability of acute localized AMP-activated protein kinase signaling to affect mitochondrial mobility within the axon, with important implications for white matter injury, axonal growth, and axonal degeneration.

Received Aug. 15, 2019; revised Apr. 8, 2020; accepted Apr. 9, 2020.

Author contributions: 0.W. and J.H.M.P. designed research; 0.W. and H.-G.K. performed research; 0.W., N.M.C.C., and H.D. analyzed data; O.W. wrote the paper; N.M.C.C., H.-G.K., H.D., and J.H.M.P. edited the paper; H.D. contributed unpublished reagents/analytic tools.

This work was supported in part by Science Foundation Ireland Research Grants 08/IN.1/B1949, 14/JPND/ $B 3077$, and 16/RC/3948, the latter of which was cofunded under the European Regional Development Fund and by FutureNeuro industry partners. This work was also supported by Innovative Medicines Initiative 2 Joint Undertaking Grant Agreement 821522; this Joint Undertaking receives support from the European Union's Horizon 2020 Research and Innovation Program and EFPIA and Parkinson's UK. We thank Dr. Jasmin Schmid, Dr. Paul Donovan, Dr. Manuela Salvucci, and Dr. Andreas U. Lindner for technical discussions and advice.

The authors declare no competing financial interests.

Correspondence should be addressed to Jochen H. M. Prehn at prehn@rcsi.ie.

https://doi.org/10.1523/JNEUROSCI.2067-19.2020

Copyright $\odot 2020$ the authors

\section{Introduction}

Neurons critically depend on mitochondria to continuously supply ATP for the maintenance of neuronal homeostasis and execution of complex processes involved in neurotransmission and synaptic plasticity (for review, see Kann and Kovács, 2007; Cheng et al., 2010). Mitochondrial bioenergetics and mobility are tightly regulated by changes in neuronal activity and energy demand (Chen and Sheng, 2013; Sajic et al., 2013). The dynamic, bidirectional nature of mitochondrial transport in polarized neurons facilitates delivery of ATP to regions of localized highenergy demand (e.g. axon growth cones to support axon/neurite growth during development, nodes of Ranvier of myelinated neurons to support action potential regeneration and propagation, 
and synaptic boutons to promote mobilization synaptic vesicles) (Morris and Hollenbeck, 1993; Mattson and Partin, 1999; Chada and Hollenbeck, 2004; Einheber et al., 2006; Pilling et al., 2006; Kang et al., 2008; Tao et al., 2014).

The axon-rich white matter of the brain comprises nearly $30 \%$ of the total brain volume (Luders et al., 2002), and localized damage in this region has been implicated in a variety of disease states, including subcortical stroke, neonatal hypoxic-ischemic encephalopathy, traumatic brain injury, and neurodegenerative disorders (Medana and Esiri, 2003; Kaur et al., 2006). The severity of local axonal injury is considered of crucial importance in determining the gravity of the neurologic deficits associated with these conditions (Blumbergs et al., 1995; Chabriat et al., 1999). Energetic deficits and resultant AMP-activated protein kinase (AMPK) activation are key features of ischemic neuronal injury (Li et al., 2007). AMPK is a key energy stress sensor that is sensitive to changes in the AMP/ATP ratio. It is intimately linked to mitochondrial bioenergetics and implicated in the determination of neuronal homeostasis by activating both energy-generating and energy-conserving pathways (Culmsee et al., 2001; Weisova et al., 2009). AMPK signaling also activates more complex cellular processes, such as autophagy and apoptosis (Concannon et al., 2010; Egan et al., 2011). Microfluidic platforms are increasingly used to study mechanisms of localized axonal injury as they facilitate the study of axons in isolation (Cusack et al., 2013; Neto et al., 2016; Zheng et al., 2019). Here we used this platform to study the effect of localized AMPK activation on mitochondrial transport within primary cortical axons. As mitochondria are the primary source of energy production, we hypothesized that their transport is affected by local changes in AMPK signaling. Indeed, direct pharmacological activation of AMPK (5-aminoimidazole-4-carboxamide riboside [AICAR], $0.1 \mathrm{~mm}$ ) at the distal axon induced a strong depression in axonal mitochondrial transport, despite the continued availability of glucose as an energy substrate. AICAR preferentially affected retrograde mitochondrial transport, resulting in an accumulation of mitochondria at the distal axon.

Disparate to the soma-rich gray matter, the axon-rich white matter region of the brain uses glial-derived lactate as its preferred substrate for ATP synthesis during periods of enhanced neurotransmission and during acute hypoglycemic events where glucose alone is insufficient to meet immediate local metabolic demands (Wender et al., 2000; Chambers et al., 2014). Indeed, numerous in vitro studies have demonstrated that lactate is sufficient to support axon function as effectively as glucose during neurotransmission and LTP (Izumi et al., 1997; Pellerin et al., 1998).

Here, we substituted glucose with a metabolic equivalent concentration of lactate at the isolated axonal compartment and found a reduction in the mean distance of mitochondrial transport events in the adjacent axon. However, the mean distance, along with the mean frequency and velocity of these mitochondrial movement events, remained stable over the $3 \mathrm{~h}$ recording period, suggesting an adaptation of axonal mitochondria to lactate as their local energy source.

Next, we induced localized nutrient deprivation at the distal axon through inhibition of lactate uptake and found a marked depression in mitochondrial transport, similar to that seen by pharmacological activation of AMPK. Antagonism of AMPK restored all parameters measured to baseline levels, confirming a role for AMPK-mediated alteration of axonal mitochondrial transport during localized energetic stress.

\section{Materials and Methods}

Materials

FBS, horse serum, MEM, insulin-transferrin-selenium, B27 supplement, bis(1,3-dibarbituric acid)-trimethine oxanol $\left[\operatorname{DiSBAC}_{4}(3)\right]$, and neurobasal medium were sourced from BioSciences. Tetramethylrhodamine methyl ester (TMRM) was obtained from Invitrogen, while AICAR was obtained from Cell Signaling Technology. 6-[(3,5-dimethyl-1H-pyrazol4-yl)methyl]-5-[[(4S)-4-hydroxy-2-isoxazolidinyl]carbonyl]-3-methyl-1(2-methylpropyl)thieno[2,3-d]pyrimidine-2,4(1H,3H)-dione (AR-C155858) was obtained from Tocris Bioscience. Formaldehyde was purchased from Thermo Fisher Scientific. All other chemicals, including compound C (CC) and sodium L-lactate, were acquired from Merck. The microfluidic devices containing $450 \mu \mathrm{m}$ microgrooves (catalog \#RD450) were sourced from Xona Microfluidics. The Amaxa nucleofector kit (catalog \#VPI-1003) was sourced from Lonza BioResearch.

Preparation and nucleofection of primary mouse neocortical neurons Primary cultures of cortical neurons were prepared from C57BL/6 mouse embryos of both sexes at embryonic day 16-18, as described previously (Concannon et al., 2010). First, the pregnant female was killed by cervical dislocation and a hysterectomy of the uterus was performed. Rapid decapitation of the embryos was conducted, and the cerebral cortices were isolated and pooled in a dissection buffer on ice $(1 \times$ PBS with $0.25 \%$ glucose and $0.3 \%$ BSA). The tissue was then incubated with $0.25 \%$ trypsin-EDTA at $37^{\circ} \mathrm{C}$ for $15 \mathrm{~min}$. After the incubation period, the trypsinization was ceased by the addition of plating media (PM). The PM is comprised of MEM with 5\% FBS, $5 \%$ horse serum, $100 \mathrm{Uml}^{-1}$ penicillin/streptomycin, $0.5 \mathrm{~mm}$ L-glutamine, and $0.45 \%$ (w/v) D-glucose. The neurons were gently dissociated using a Pasteur pipette, and after centrifugation at $1500 \mathrm{rpm}$ for $3 \mathrm{~min}$, the medium containing trypsin was aspirated. The remaining cell pellet was resuspended in fresh PM.

The primary cortical neurons were then transfected with mitochondrial-targeted GFP (mito-GFP; pAcGFP1-Mito, Clontech) using AMAXA nucleofection technology (Lonza BioResearch). The mito-GFP plasmid comprises a viral vector containing fusion construct of a mitochondrial targeting sequence from subunit VIII of human cytochrome $c$ oxidase and GFP from Aequorea coerulescens (AcGFP1); $\sim 5 \times 10^{6}$ cells were collected by centrifugation at $1500 \mathrm{rpm}$ for $3 \mathrm{~min}$. The supernatant was aspirated, and $100 \mu \mathrm{l}$ of specific nucleofector solution was added to resuspend the pellet of cells; $3 \mu \mathrm{g}$ of mito-GFP plasmid was added to the suspension and transferred into an Amaxa-certified $100 \mu \mathrm{l}$ aluminum electrode cuvette for nucleofection, as per the manufacturer's instructions.

WillCo dishes (WillCo Wells) of $35 \mathrm{~mm}$ diameter were assembled, sterilized, and coated with $0.5 \mathrm{mg} / \mathrm{ml}$ poly-L-lysine for $24-48 \mathrm{~h}$ before use. The microfluidic devices were gently placed onto the coated WillCo dishes, and the 4 wells of the device were rinsed with PM before cell loading. Cells were seeded at a density of $5 \times 10^{6}$ cells $/ \mathrm{ml}$ in PM and insulin-transferrin-selenium supplement, into the 2 wells on one side of the device, allowing them to flow into their connecting microfluidic compartment while the second compartment was filled with PM only. The cultures were maintained in a sterile humidified incubator at $37^{\circ} \mathrm{C}$, $5 \% \mathrm{CO}_{2}$ for 9-10 DIV before experimentation. After $24 \mathrm{~h}$, the PM was exchanged with $50 \%$ feeding medium (neurobasal medium containing $100 \mathrm{Uml}^{-1}$ penicillin/streptomycin, 2\% B27, and $0.5 \mathrm{~mm}$ L-glutamine) and 50\% PM with additional cytosine arabinofuranoside (600 nM). At DIV 2, the medium was exchanged for complete feeding medium.

All animal work was performed with ethics approval by the RCSI Research Ethics Committee (REC1335) and under a license granted by the Irish Department of Health and Children (B100/4442).

\section{Immunocytochemistry}

At DIV 10, the microfluidic device was carefully removed and the media was quickly replaced with $3 \%$ formaldehyde diluted in prewarmed cytoskeletal buffer (in mM as follows: 10 PIPES, pH 6.8, $300 \mathrm{NaCl}, 10$ EGTA, 10 glucose, $10 \mathrm{MgCl}_{2}$ ), for $12 \mathrm{~min}$ at $37^{\circ} \mathrm{C}$. The fixed cells were then permeabilized with $0.1 \%(\mathrm{w} / \mathrm{v})$ Triton X-100 and blocked with $0.3 \%(\mathrm{w} / \mathrm{v})$ Triton X-100 and 5\% (v/v) horse serum in PBS. After overnight incubation at $4^{\circ} \mathrm{C}$ in primary antibody (1:500 dilution in $\mathrm{PBS} / 0.3 \%(\mathrm{w} / \mathrm{v})$ 
Triton X-100/3\% (v/v) horse serum), the cells were exposed to $3 \times 5 \mathrm{~min}$ washes in ice-cold PBS. The primary antibodies used were goat polyclonal to MAP2 (1:2000; RRID:AB_448205, Abcam), mouse monoclonal to phospho-PHF-Tau (1:500; RRID:AB_223651, Thermo Fisher Scientific), and rabbit polyclonal to AMPK $\alpha$ (RRID:AB_561524, Cell Signaling Technology). The cultures were then incubated with respective speciesspecific AlexaFluor-488/-536/-568-coupled secondary antibodies (RRID: AB_2535788/AB_2534017/AB_10584487, respectively, Invitrogen) at a dilution of 1:500 for $1 \mathrm{~h}$ at room temperature. Hoechst $33258(1 \mu \mathrm{g} / \mathrm{ml}$, Sigma Millipore) was used to visualize nuclei by excitation at $405 \mathrm{~nm}$. Immunofluorescence was visualized at $20 \times$ magnification (Plan-Apochromat $20 \times 1$ $0.8 \mathrm{NA}$ ) using an LSM 710 confocal microscope (Carl Zeiss), and images were processed using ImageJ (National Institutes of Health).

\section{Protein extraction and Western blotting}

Primary cortical neurons were maintained at $37^{\circ} \mathrm{C}, 5 \% \mathrm{CO}_{2}$ for 910 DIV before acute treatment $(30-120 \mathrm{~min})$ with lactate in the presence or absence of AR-C155858. At specified time points, the cells were washed in PBS and lysed on ice with a lysis buffer containing $0.5 \mathrm{~mm}$ Tris- $\mathrm{HCl}(\mathrm{pH} 6.8), 2 \%$ SDS (w/v), and protease inhibitor cocktails (Merck). Protein concentrations were determined using a micro BCA protein assay kit (Thermo Fisher Scientific). An equal amount of proteins $(20 \mu \mathrm{g})$ were diluted with Laemmli loading buffer and were separated on 10\% SDS-polyacrylamide gels. Proteins were then transferred to nitrocellulose membranes in transfer buffer $(25 \mathrm{~mm}$ Tris, $192 \mathrm{~mm}$ glycine, $20 \%$ methanol (v/v), and $0.01 \%$ SDS) at $18 \mathrm{~V}$ for $90 \mathrm{~min}$. The nitrocellulose

membranes were then blocked with $5 \%$ nonfat dry milk in TBS (15 mM Tris-HCl, pH 7.5, $200 \mathrm{~mm} \mathrm{NaCl}$ ) containing 0.1\% Tween 20 (TBS-T), at room temperature for $1 \mathrm{~h}$. They were incubated with primary antibodies (1:1000 dilution); rabbit monoclonal to phospho-AMPK $\alpha$ (Thr172) (RRID:AB_331250, Cell Signaling Technology) and mouse monoclonal $\beta$-actin (RRID:AB_476744, Sigma Millipore) overnight at $4^{\circ} \mathrm{C}$. The membranes were then washed with TBS-T before incubation with mouse (RRID:AB_90456, Millipore) or rabbit (RRID:AB_90264, Millipore) IgG HRP-conjugated secondary antibodies (1:1000 dilution) in 5\% milk for $1 \mathrm{~h}$. Proteins were then detected using enhanced chemiluminescence detection reagent (GE Healthcare) at 12-bit dynamic range using a Fuji LAS 4000 CCD system (Fujifilm UK).

In order to assess the levels of total AMPK $\alpha$, which has an equivalent molecular weight to its phosphorylated form, the membranes were first stripped with Restore Western Blot Stripping Buffer (Thermo Fisher Scientific) according to the manufacturer's instructions. They were then reprobed with anti-rabbit secondary antibody (RRID:AB_90264) to confirm removal of the primary antibody against phospho-AMPK before incubation overnight at $4^{\circ} \mathrm{C}$ in rabbit polyclonal antibody to AMPK $\alpha$ (RRID:AB_330331, Cell Signaling Technology), at a dilution of 1:1000 in $5 \%$ milk. The membranes were then washed with TBS-T and incubated once more in rabbit (RRID:AB_90264) IgG HRP-conjugated secondary antibody (1:1000 dilution) in 5\% milk for $1 \mathrm{~h}$. AMPK $\alpha$ levels were then detected as described above. The stripped blots were also reprobed for their loading control, $\beta$-actin, as described above.

\section{Live cell imaging}

At 9-10 DIV, extensive axon projections of a subset of the cultured cortical neurons were present, some of which had grown through the microgrooves (length: $450 \mu \mathrm{m}$ ) of the microfluidic device and now occupied the media-filled compartment. This compartment, now axon-rich, is referred to in this manuscript as the "axonal compartment", whereas the compartment containing the soma and dendrites of this subset of neurons is referred to as the "somatodendritic compartment". Culture media was replaced with prewarmed $\left(37^{\circ} \mathrm{C}\right)$ experimental buffer containing the following (in mM): $120 \mathrm{NaCl}, 3.5 \mathrm{KCl}, 0.4 \mathrm{KH}_{2} \mathrm{PO}_{4}, 20 \mathrm{HEPES}, 5$ $\mathrm{NaHCO}_{3}, 1.2 \mathrm{Na}_{2} \mathrm{SO}_{4}$, and $1.2 \mathrm{CaCl}_{2}, \mathrm{pH}$ 7.4. Depending on the energy substrate required for the experiment, $15 \mathrm{~mm}$ glucose or $30 \mathrm{~mm}$ lactate was also added to the buffer. A hydrostatic pressure gradient was established across the device by manipulating the volumes in the somatodendritic and axonal compartments, effectively allowing the isolation of drug treatment to the compartment with the lower volume of experimental buffer (Taylor et al., 2003). For all experiments, an equal volume of drug (AICAR \pm CC or AR-C155858 \pm CC) or experimental buffer (control) was added to the compartment to rule out any confounding effects of changes in fluidic pressure on cellular function and integrity.

Mineral oil was placed on top of all four wells of the device to prevent evaporation during imaging. The WillCo dish was transferred to the stage of an LSM 710 confocal microscope (Carl Zeiss), which was encased in a thermostatically regulated chamber, maintained at $37^{\circ} \mathrm{C}$.

Mitochondrial tracking. Using the EC Plan-Neofluar $40 \times / 1.30$ Oil DIC M27 objective (Carl Zeiss), the FOV was positioned at the end of the microgrooves, closest to the axonal compartment. At this magnification, $\sim 354 \mu \mathrm{m}$ of the microgroove length was within the FOV. Using the epifluorescence light source, a microgroove containing one axon (in rare cases, two axons) expressing GFP-positive mitochondria was selected for imaging. Mito-GFP was excited at $488 \mathrm{~nm}$ wavelength, and the emission was collected in the range of $500-550 \mathrm{~nm}$ from an optical section set to $10 \mu \mathrm{m}$ (FWHM). Time-lapse confocal imaging was conducted at a rate of $0.25 \mathrm{~Hz}$ for $3 \mathrm{~h}$ with a hardware based autofocus unit keeping the focus stable (Definite Focus, Carl Zeiss). After 30 min baseline recording, experimental buffer (control) or drug cocktail (AICAR \pm CC, AR-C155858 \pm CC) was added to the axonal or somatodendritic compartment. For experiments involving axonal lactate, 60 min baseline recording was obtained to ensure equilibration of the axons in the lactate-enriched buffer and stabilization of mitochondrial transport dynamics before drug addition.

Mitochondrial counts at the distal axon. In order to monitor the number of GFP-positive mitochondria contained within the distal axon directly exposed to the treatment, confocal images were taken at this region at $40 \times$ magnification using the same excitation/emission parameters as before. Mitochondrial membrane potential was also recorded in this region by the addition of $20 \mathrm{~nm}$ (nonquenching concentration) TMRM to the experimental buffer for $20 \mathrm{~min}$ before confocal imaging. TMRM was excited at $561 \mathrm{~nm}$, and emission was collected in the range of 560-700 nm. Confocal images of mito-GFP and TMRM fluorescence were taken at $10 \mathrm{~min}$ intervals for $30 \mathrm{~min}$ before, and $2 \mathrm{~h}$ after the addition of the pharmacological agent(s).

As voltage-sensitive probes, such as TMRM, are also sensitive to changes in the plasma membrane potential, this experiment was repeated in the presence of the plasma membrane fluorescent reporter, $\operatorname{DiSBAC}_{4}(3) .1 \mu \mathrm{M} \operatorname{DiSBAC}_{4}(3)$ was added to the experimental buffer $20 \mathrm{~min}$ before imaging and remained in the buffer for the duration of the experiment. $\operatorname{DiSBAC}_{4}(3)$ was excited at $488 \mathrm{~nm}$, and the emission was collected in the range of $490-560 \mathrm{~nm}$. Confocal images were taken as described above.

\section{Image processing and data analysis}

Mitochondrial tracking analysis. The time series of confocal images were processed using ImageJ to generate kymographs representing the position of the mitochondria along the axon ( $x$ axis) over time ( $y$ axis). The kymographs were subdivided into six sections, each representing 450 frames ( $30 \mathrm{~min}$ recording) of the experiment. These kymographs were then loaded into MetaMorph Software version 7.5 (Universal Imaging) for analysis. Each diagonal line on the kymograph corresponds to a "moving event", the direction of which is defined by the angle of the line. The diagonal lines, which showed a distinct beginning and endpoint, were traced using the "region tool". The slope of the lines corresponds to the velocity of the moving events, whereas the difference in the start and endpoint of the $y$ coordinates of the line corresponds to the distance traveled. A mitochondrion was defined as mobile if its position changed over at least three consecutive frames. A macro was created within Microsoft Excel (Microsoft) to extract the data collected from MetaMorph. The moving events were categorized based on their directional movement (anterograde and retrograde), and the velocity and distance of these events over time were analyzed.

Analysis of mitochondria at the distal axon. Mito-GFP-positive mitochondria in the axonal compartment were counted using ImageJ. First, the background was removed from the series of images, and the median filter was applied. A threshold was set to include all mito-GFP-positive 
mitochondria, and the mitochondrial counts over time were extracted. Mitochondrial counts $2 \mathrm{~h}$ after treatment (T120) were normalized to pretreatment counts (T0) within each experiment.

TMRM fluorescence was used as an indicator of the mitochondrial membrane potential in all axons (both transfected and nontransfected) directly exposed to drug treatment. After background correction, a threshold was applied using ImageJ to include all TMRM-positive mitochondria, and the mean fluorescence intensity (sum of intensities of all pixels above threshold normalized to the number of pixels above threshold) was calculated. Similar background correction and thresholding were applied to $\mathrm{DiSBAC}_{4}(3)$ images to calculate its mean fluorescence intensity over time, indicative of changes in the plasma membrane potential in axons directly exposed to drug treatment. For each experiment, measurements were normalized to pretreatment values (T0) and the change in TMRM fluorescence after $2 \mathrm{~h}$ of treatment (T120) with $\mathrm{AICAR} \pm \mathrm{CC}$ was compared.

Analysis of stationary mitochondria. Stationary mitochondria were classified on each kymograph by identifying vertical lines using a MATLAB (R2017a) self-written function, the code for which is available at https:/github.com/niamhconno/Watters-et-al-2020-jNeurosci. Mitochondria were identified as "potentially stationary" if their centerpoints moved $< \pm 3.5 \mu \mathrm{m}$ over the $30 \mathrm{~min}$ period. These assignments were then confirmed or rejected via user input.

\section{Statistics}

All data obtained from kymographs are expressed as mean \pm SEM. Unless otherwise stated, each set of experiments contains $n \geq 6$ experiments, from at least three independent culture preparations. All datasets complied from time-lapse confocal imaging experiments were first normalized against the $30 \mathrm{~min}$ baseline recording interval within each experiment. Labeling on the $x$ axis is taken as the "final time point" of the $30 \mathrm{~min}$ recording interval, with -30 and 0 representing the $0-30$ and 30-60 min interval before drug treatment, respectively, and 30 representing the first $30 \mathrm{~min}$ interval after drug addition, and so on. Statistical analyses were performed with GraphPad Prism software 6.0 (GraphPad Software). A repeated-measures ANOVA and Dunnett's multiple-comparison post hoc test were used to compare the pooled data from each time interval with their corresponding baseline values (T0). Because of the high-frequency imaging acquisition set for these experiments $(0.25 \mathrm{~Hz})$, alterations in control conditions over time were evident in some cases. For this reason, a strict statistical significance threshold of $p$ values $\leq 0.01$ was set, denoted by asterisk.

A one-way ANOVA and a Dunnett's multiple-comparison post hoc test was used to analyze statistical differences between treatment groups (see Fig. 4CII).

Western blot analysis of changes in pAMPK and total AMPK levels during ARC-155858 treatment (see Fig. 6A) were also analyzed using a one-way ANOVA and Dunnett's multiple-comparison post hoc test. The degrees of freedom (df1, df2), $F$ value, and exact $p$ value generated from ANOVA statistical tests are also reported for each significant comparison highlighted in this manuscript.

Each data point in Figure $4 B$ represents the total mitochondrial count within each FOV before (T0) and $2 \mathrm{~h}$ post (T120) drug treatment at the distal axon. A paired $t$ test was performed to analyze the change mitochondrial counts per FOV $2 \mathrm{~h}$ after drug addition. Western blot analysis and comparison of baseline mitochondrial transport parameters between experiments where glucose or lactate was the energy substrate at the distal axons were performed using an unpaired $t$ test (see Fig. 5). The df, $t$ value, and exact $p$ value generated from $t$ tests are also reported for each significant comparison highlighted in this manuscript.

\section{Results}

AMPK is expressed throughout primary cortical neurons, including in dendritic and axonal processes

We set out to establish a platform of axonal isolation in microfluidic devices that allowed us to explore the effect of localized treatment of specific neuronal compartments (somatodendritic vs axonal) on mitochondrial mobility within the axons. In order to monitor mitochondrial movement, we first expressed mitoGFP plasmid using AMAXA nucleofection before seeding. After 9-10 DIV, confocal microscopy (20× magnification) was used to examine the expression of the mito-GFP protein in these cells. GFP-tagged mitochondria were clearly visible within the somatodendritic regions and axons of transfected cells, which projected through the microgroove of the microfluidic device (Fig. 1A). As not all cells were transfected, this technique allowed us to clearly distinguish mitochondria within one (or two) axon(s) extending through a single microgroove (Fig. $1 A, B$ ). TMRM, a cell-permeant cationic dye that accumulates within active mitochondria with intact mitochondrial membrane potentials, colocalized with mito-GFP (Fig. 1B). Similar TMRM fluorescence was found in the mitochondria of transfected and nontransfected cells, suggesting that mito-GFP expression did not affect mitochondrial membrane potential integrity.

Immunocytochemistry was then conducted to confirm AMPK expression in the axons of mature cortical neurons in vitro (Turnley et al., 1999). First, we validated that the neuronal processes which extended through the microgrooves and into the second compartment were primarily axonal, as prominent Tau (PHF-Tau) expression was found. The somatodendritic compartment also contains Tau staining, highlighting the axons that did not project through the microgrooves. The dendritic processes (MAP-2 positive) of all the neurons were visible mainly in the somatodendritic compartment of the microfluidic device. Coimmunolabeling with the anti-AMPK antibody confirmed the global expression of AMPK throughout the neuron, including the axonal processes (Fig. 1C).

\section{AMPK activation at the somatodendritic region does not affect mitochondrial transport in the spatially isolated axon segment}

Previous work in our laboratory has identified that treatment of cortical neurons with the pharmacological agonist of AMPK, AICAR, at a concentration of $0.1 \mathrm{~mm}$, rapidly induces robust phosphorylation of AMPK to its active state for up to $24 \mathrm{~h}$ after treatment, without compromising cell integrity (Anilkumar et al., 2013). We and others have shown that this concentration of AICAR is also sufficient to induce prolonged neuroprotection against metabolic, excitotoxic, and oxidative insults within a therapeutic window of $24 \mathrm{~h}$ (Culmsee et al., 2001; Anilkumar et al., 2013). Both pharmacological inhibition (CC, $10 \mu \mathrm{M}$ ) and gene silencing of AMPK (AMPK-antisense oligonucleotide and AMPK siRNA) remove AICAR-induced neuroprotection, confirming that this preconditioning effect is indeed mediated by AMPK signaling (Culmsee et al., 2001; Anilkumar et al., 2013). As mitochondrial bioenergetics play an essential role in the maintenance of energy homeostasis, we investigated whether neuroprotective levels of the AMPK energetic stress signal would locally influence mitochondrial transport kinetics and distribution.

First, we investigated whether AMPK activation isolated to the somatodendritic region of the neuron could influence mitochondrial transport dynamics within the spatially isolated axon segment. The experimental buffer volumes were manipulated across the microfluidic device to favor restriction of AICAR to the somatodendritic compartment, while imaging was conducted at the distal microgroove segment measuring $\sim 354 \mu \mathrm{m}$ (FOV; Fig. 2A). The section of the microgroove was chosen next to the opening into the axonal compartment to prevent inadvertent imaging of mitochondria within dendrites, which may partially extend into the initial section of the microgroove (Taylor et al., 2005). 
A

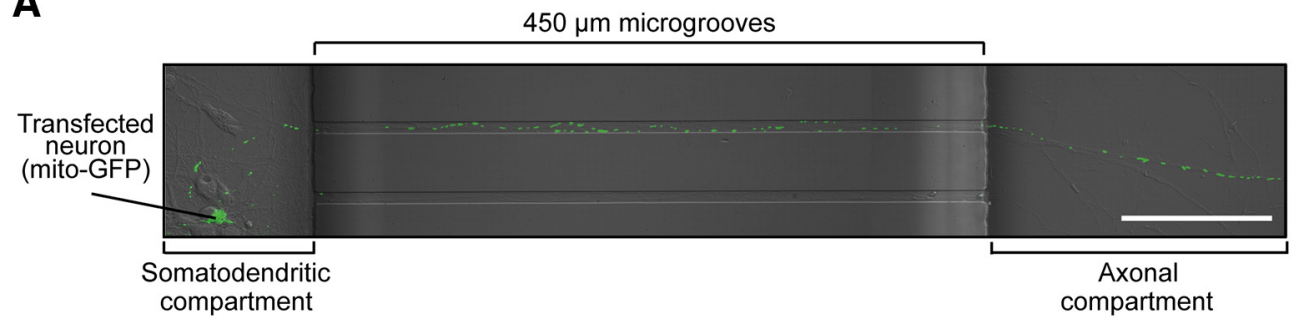

B

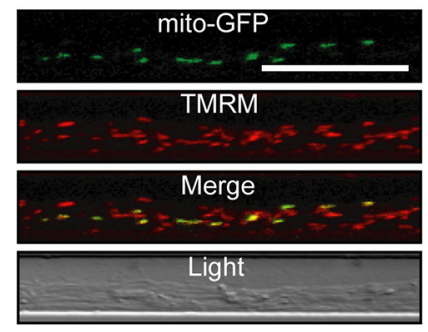

C
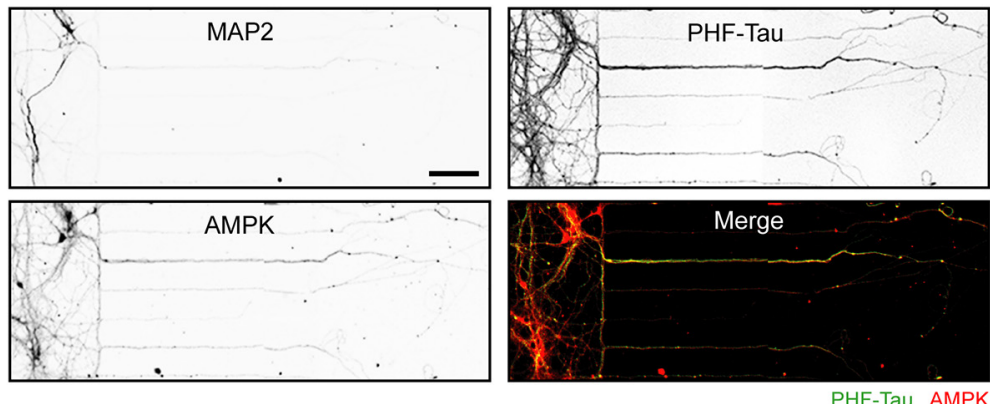

Figure 1. AMPK is expressed in the soma and throughout the dendritic and axonal processes of mouse primary cortical neurons. $A$, Representative confocal image $(20 \times)$ of a primary cortical neuron (transfected with mito-GFP before plating) at DIV 10 with its process extended through a microgroove (450 $\mu \mathrm{m})$ of the device and into the second compartment (axonal compartment). Scale bar, $100 \mu \mathrm{m}$. B, Colocalization of GFP-positive mitochondria with TMRM, a fluorescent indicator of the mitochondrial membrane potential confirmed the viability of transfected mitochondria. Scale bar, $20 \mu \mathrm{m}$. C, Immunocytochemical analysis confirmed that many of the primary cortical neurons cultured for 10 DIV within the microfluidic device extend their axonal processes (PHF-Tau-positive) through the microgrooves of the device. Limited dendritic processes (MAP2-positive) extend into the axonal compartment of the device. Inverted grayscale images for each antibody are shown in panels 1-3. The "Merge" image (panel 4) shows that AMPK staining (green) colocalizes PHF-Tau (red), indicating the presence of AMPK in the axons. Scale bar, $100 \mu \mathrm{m}$.

Mitochondrial dynamics and distribution along the axon were examined under baseline conditions to ensure that manipulation of hydrostatic pressure across the device had no adverse effect. The mean number of mitochondria in the imaged axonal section from this group of experiments was $58.7 \pm 3.3$ (16 mitochondria/100 $\mu \mathrm{m}$ axon) comparable to previous studies (Chang et al., 2006). Mitochondria were relatively evenly distributed throughout the axonal segment (proximal: $33.7 \pm 1.0 \%$, central: $34.1 \pm 0.7 \%$, distal: $32.5 \pm 1.2 \%$; $n=46$ experiments) with the percentage of stationary mitochondria $(45.3 \pm 2.7 \%)$ similar to that noted in previous studies in primary neurons (Ligon and Steward, 2000; Quintanilla et al., 2012). Mitochondrial morphology was also examined with the mean major axis length reported as $2.1 \pm 0.06 \mu \mathrm{m}$ again in line with previous measurements (Dickey and Strack, 2011; Quintanilla et al., 2012), suggesting healthy, functioning mitochondrial transport systems in these mito-GFP-transfected neurons. Thus, with AMPK expression established within axons on the microfluidic platform, and mitochondrial morphology, distribution, and mobility following mito-GFP nucleofection confirmed to lie within physiologically relevant ranges, we next monitored real-time effects of AMPK signaling on mitochondrial transport.

Mitochondrial transport was measured in the axon segment at a rate of 1 frame every $4 \mathrm{~s}(0.25 \mathrm{~Hz})$. After $30 \mathrm{~min}$ baseline recording (T0), $0.1 \mathrm{~mm}$ AICAR or $10 \mu \mathrm{M}$ CC was added to the somatodendritic compartment while mitochondrial mobility in the microfluidically isolated axonal region (FOV) was monitored for a further $2.5 \mathrm{~h}$ (Fig. 2AII). Kymographs were generated from the time series of confocal images as a graphical representation of mitochondrial movement over time. Representative kymographs ( $5 \mathrm{~min}$ ) during baseline recording (T0) and $2 \mathrm{~h}$ after treatment (T120) are shown in Figure 2B.

Neither pharmacological activation nor inhibition of AMPK at the somatodendritic compartment, with AICAR and CC, respectively, altered the frequency of mitochondrial movement events in either direction within the spatially isolated axon segment (Fig. 2C), suggesting no perturbation of the balance of bidirectional mitochondrial transport frequency. Analysis of the baseline mitochondrial transport kinetics of the mobile mitochondria in this axon segment recorded a mean mitochondrial velocity of $0.34 \pm 0.01 \mu \mathrm{m} / \mathrm{s}$, while mobile mitochondria traveled a mean distance of $11.86 \pm 0.75 \mu \mathrm{m}$ per movement event. Addition of AICAR or CC to the somatodendritic region did not alter the velocity or distance of mobile mitochondria, in either direction, over the $2.5 \mathrm{~h}$ treatment period (Fig. 2D,E). Together, these results suggest that localized modulation of AMPK activity at the soma does not influence mitochondrial transport in the spatially isolated axon segment.

\section{Local AMPK activation in the distal axon reduces the mean frequency, velocity, and distance of axonal mitochondrial transport}

Next, we investigated whether mild activation of AMPK signaling isolated to the distal axon would affect mitochondrial transport in the adjacent axon segment. First, the experimental buffer volumes were manipulated across the microfluidic device to favor restriction of AICAR to the axonal compartment, while imaging was conducted on the same microgroove segment (FOV) as before (Fig. 3A).

Mitochondrial dynamics and distribution along the axon were examined once more under baseline conditions to ensure that manipulation of hydrostatic pressure across the device had no adverse effect. The average number of mitochondria contained within the axon of transfected cells within this section was $57.64 \pm 3.9$, averaging at 16 mitochondria/100 $\mu \mathrm{m}$ axon. Even mitochondrial distribution was also noted in this experimental setup (proximal, $34.8 \pm 0.9 \%$; central, $33.4 \pm 0.7 \%$; distal, $31.6 \pm$ $0.9 \% ; n=50$ experiments; data not shown). The mean percentage 
Al

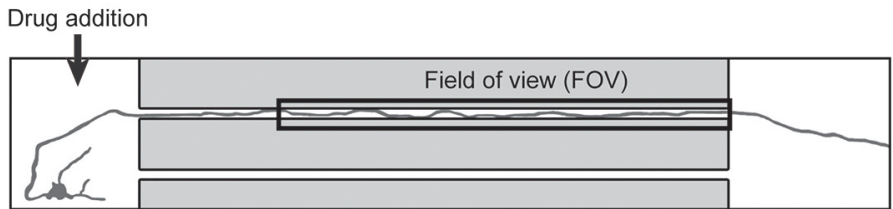

All

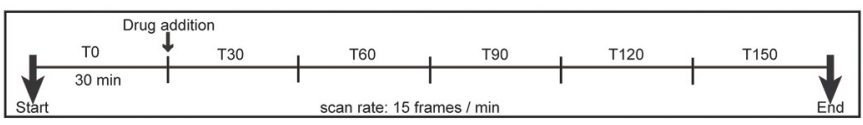

BI

C
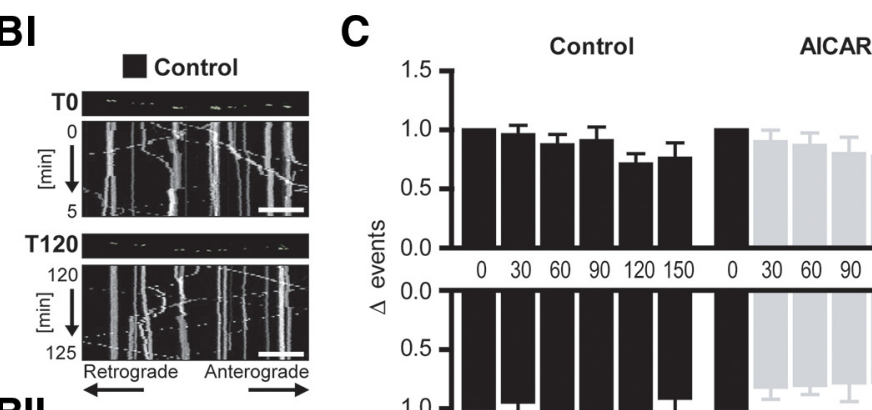

BII
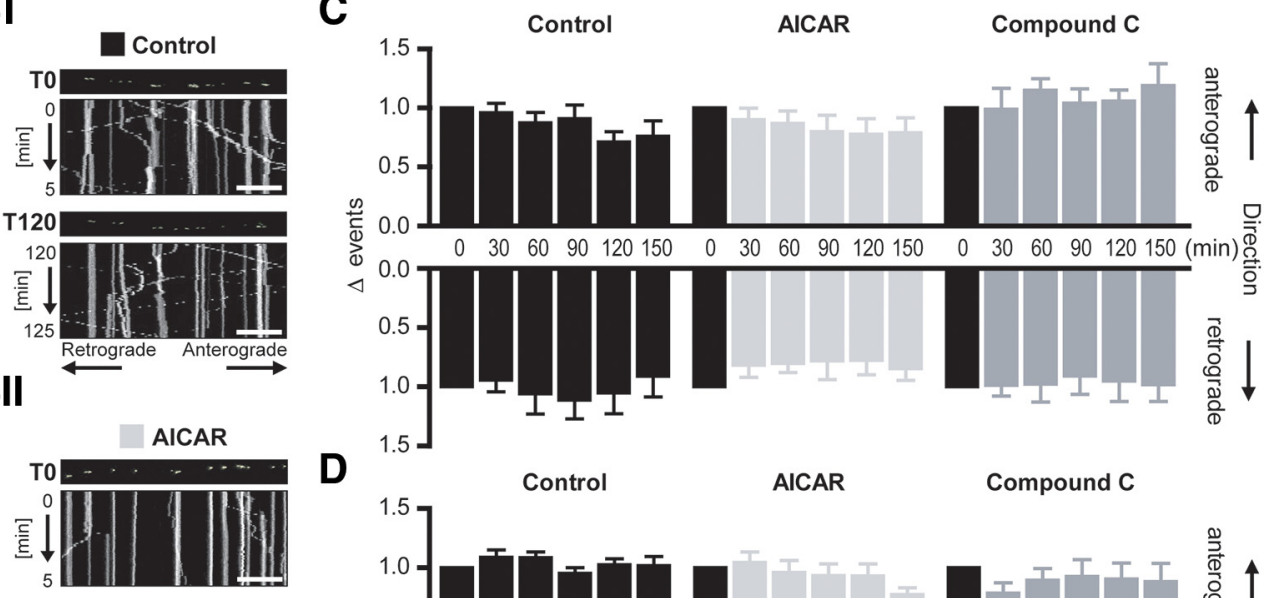

D

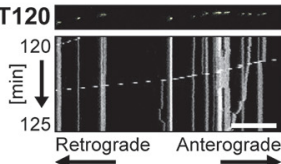

BIII
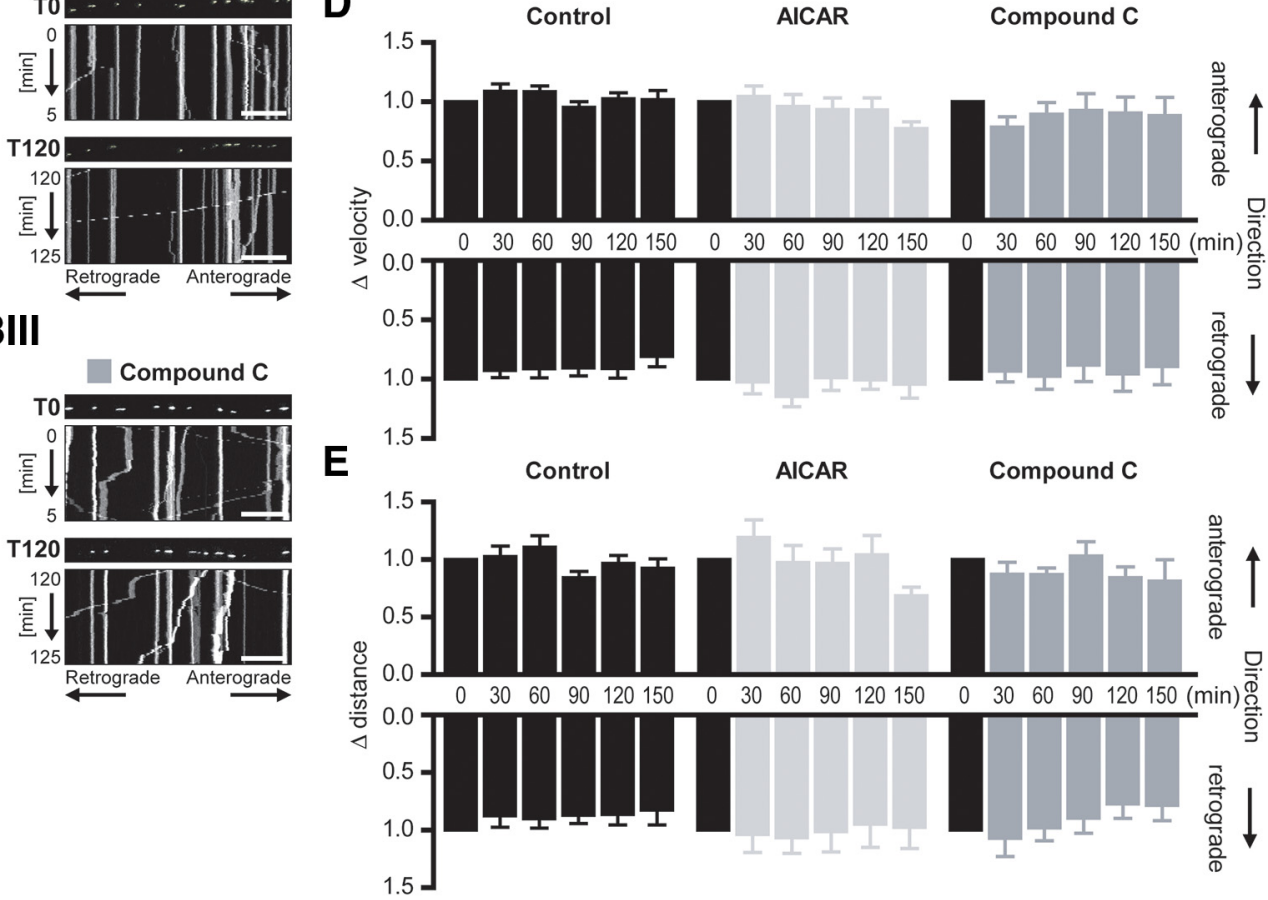

Figure 2. AMPK activation at the somatodendritic region did not alter mitochondrial mobility within the spatially isolated axon segment. $\boldsymbol{A}$, Schematic of experimental design and time course. Al, Drug addition occurred at the somatodendritic compartment (indicated by the arrow) while mitochondrial mobility was measured in the spatially isolated distal axon segment contained within the microgroove of the microfluidic device (FOV). All, Mito-GFP fluorescence in this axon segment was imaged at a rate of $15 \mathrm{frames} / \mathrm{min}(0.25 \mathrm{~Hz})$ for $3 \mathrm{~h}$, with drug addition to the somatodendritic compartment after $30 \mathrm{~min}$ (T0) baseline recording. $\boldsymbol{B}$, Representative kymographs of mitochondrial mobility in the axon segment before (T0) and $2 \mathrm{~h}$ (T120) after treatment at the somatodendritic compartment with (BI) experimental buffer (Control), (BII) $0.1 \mathrm{~mm}$ AICAR, or (BIII) $10 \mu \mathrm{m}$ CC. Mito-GFP-tagged mitochondria can be visualized in the still image shown above each kymograph (first image of corresponding time interval). Scale bars, $20 \mu \mathrm{m}$. C, The frequency of both anterograde and retrograde mitochondrial moving events within the axon segment remained unaltered by AMPK activation (AICAR) or inhibition (CC) at the somatodendritic region. Mean velocity (D) and distance $(\boldsymbol{E})$ of mitochondrial movements in both anterograde and retrograde directions remained relatively constant within the spatially isolated distal axon segment during AICAR or CC treatment at the somatodendritic region. Repeated-measures ANOVA, Dunnett's multiple-comparison post-test. $n \geq 6$ experiments from at least four independent culture preparations.

of stationary mitochondria during baseline recordings was $42.2 \pm 2.1 \%$, similar to that seen under the previous experimental conditions. We also observed an average mitochondrial length of $2.43 \pm 0.27 \mu \mathrm{m}$, in line with previous measurements recorded in this study and others (Dickey and Strack, 2011; Quintanilla et al., 2012). All of the aforementioned baseline parameters did not differ significantly from that measured on reversal of the hydrostatic pressure gradient in the previous experimental setup, eliminating the hydrostatic pressure gradient as a confounding variable in these experiments.

After 30 min baseline recording, $0.1 \mathrm{~mm}$ AICAR was added to the axonal compartment and mitochondrial mobility was monitored in the adjacent axonal region (FOV) for a further $2.5 \mathrm{~h}$ (Fig. 3A). Representative kymographs (5 min) during baseline 
A

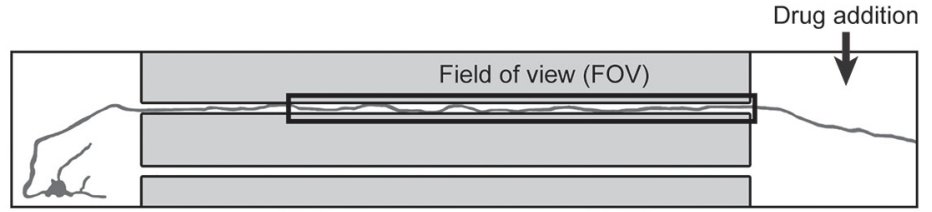

BI
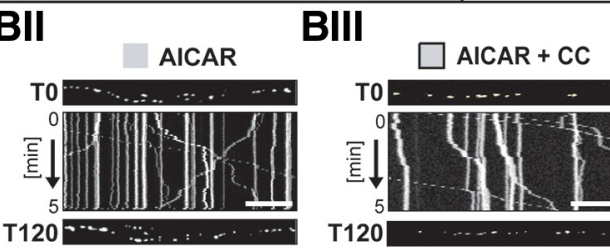

\section{BIV}
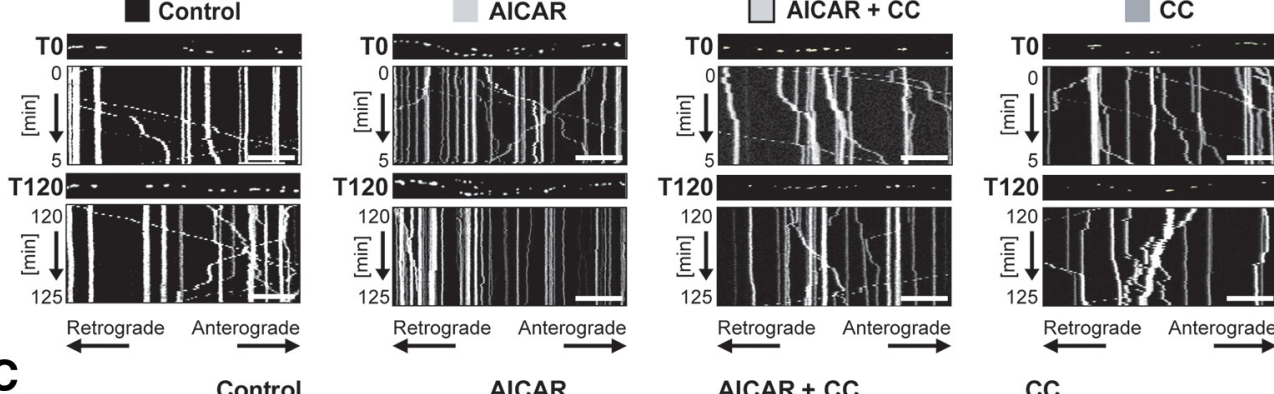

T120

C

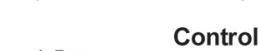

AICAR
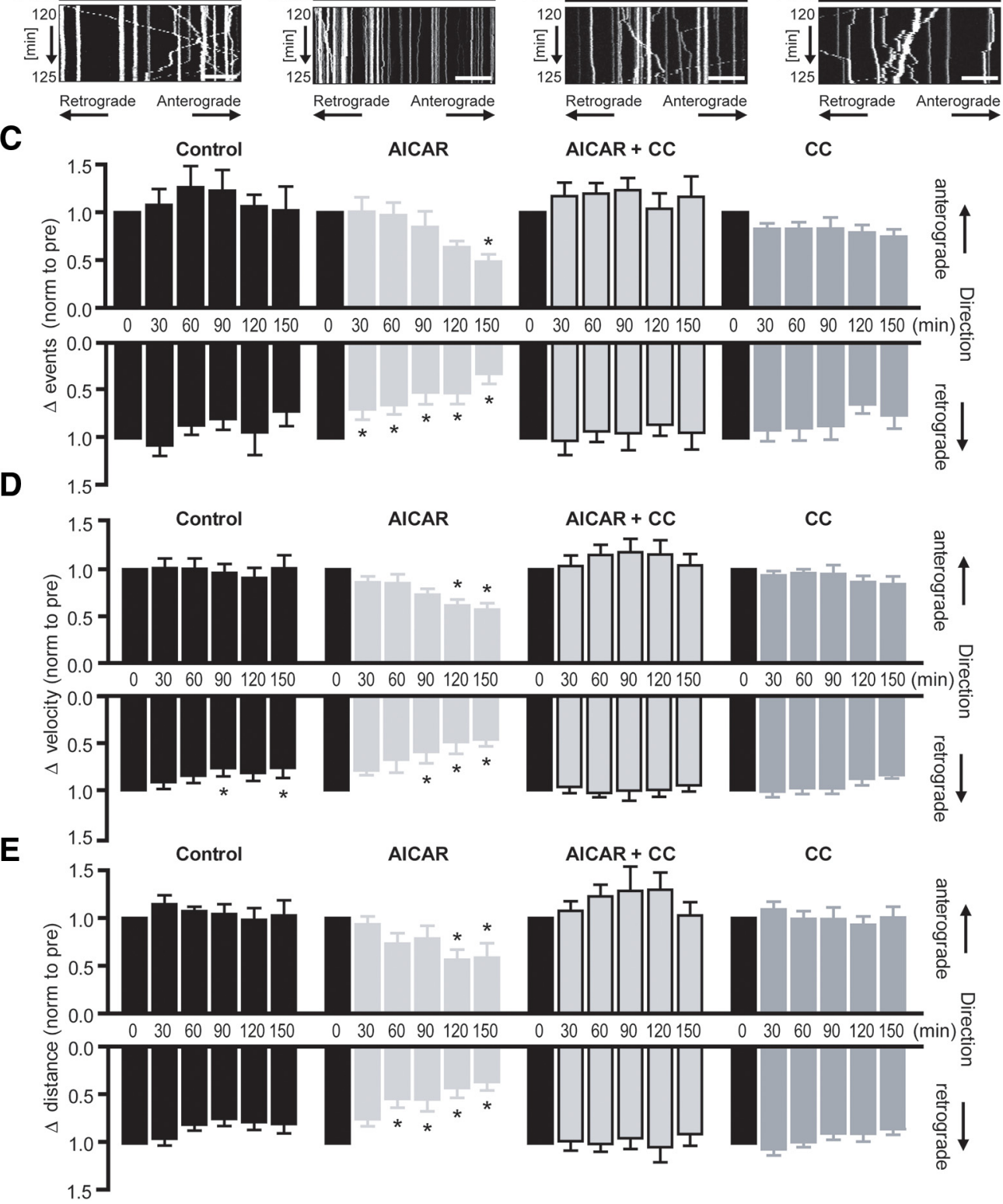

Figure 3. Localized AMPK activation at the distal axon reduces the frequency velocity and distance of mitochondrial transport in the adjacent axon segment. $A$, Schematic of experimental design. Mitochondrial mobility was measured in the axon segment contained in the microgroove (FOV) adjacent to the area of drug addition (axonal compartment). Mito-GFP fluorescence in this axon segment was imaged as before (see Fig. 2AII). B, Representative kymographs of mitochondrial mobility in the axon segment FOV during baseline recording (T0) and $2 \mathrm{~h}$ (T120) after treatment with (BI) experimental buffer (Control), (BII) $0.1 \mathrm{~mm} \mathrm{AICAR,} \mathrm{(BII)} \mathrm{AICAR} \mathrm{and} 10 \mu \mathrm{m} \mathrm{CC}$, or (BIV) CC alone, at the distal axon. Mito-GFP-tagged mitochondria can be visualized in the still image shown above each kymograph (first image of corresponding time interval). Scale bar, $20 \mu \mathrm{m}$. C, Mitochondrial transport kinetics was extracted from the kymographs, and the mean velocity and distance of mitochondrial movement events were normalized to their respective pretreatment values (TO). AICAR (0.1 mM) provoked a rapid decrease in the frequency of retrograde mitochondrial movements (T30) with a secondary depression in anterograde movement frequency at a later time point (T150). D, Under control conditions, a mild depression in retrograde transport velocity was observed. However, AICAR treatment induced a stronger, more sustained depression of retrograde mitochondrial transport velocity (T90), with a similar effect evident in anterograde transport at a later time point (T120). $\boldsymbol{E}$, The mean distance of retrograde mitochondrial transport was rapidly reduced (T60) up AICAR treatment at the distal axon, with the mean distance of anterograde transport affected at a much later time point (T120). Antagonism of AMPK activity with CC (10 $\mu \mathrm{M})$ reversed all AICAR-mediated effects on mitochondrial transport. ${ }^{*} p \leq 0.01$ (repeated-measures ANOVA with Dunnett's multiple-comparison post hoc test). $n \geq 6$ experiments from at least three independent culture preparations. 
recording (T0) and $2 \mathrm{~h}$ after treatment (T120) are shown in Figure $3 B$. Kymograph analyses reported that the mean number of mitochondrial moving events within the imaged axon segment under baseline conditions was $282 \pm 18.8$, with $52.0 \pm 1.7 \%$ and $47.9 \pm 1.7 \%$ of these movements occurring in the anterograde and retrograde directions, respectively (data not shown).

Treatment of the axonal compartment with AICAR induced a progressive depression in the frequency of retrograde mitochondrial movements, reaching statistical significance $30 \mathrm{~min}$ after treatment (Fig. 3C; T30: $51.9 \pm 12.6 \%, F_{(5,25)}=13.89, p=$ $0.008, n=6$ ), whereas a similar depressive effect was seen on anterograde mitochondrial transport at a much later time point (T150: $48.3 \pm 7.7 \%, F_{(5,25)}=5.726, p=0.0021, n=6$ ).

We next evaluated whether AMPK activation had an effect on the kinetics of mitochondrial transport, focusing on the velocity and distance traveled by mobile mitochondria during each movement event. Under baseline conditions (T0), the mean velocity of the movement of GFP-positive mobile mitochondria was $0.39 \pm 0.01 \mu \mathrm{m} / \mathrm{s}$, comparable to published values (Morris and Hollenbeck, 1995; Ligon and Steward, 2000). Treatment of the axonal compartment with $0.1 \mathrm{~mm}$ AICAR induced a significant decrease in mitochondrial transport velocity affecting retrograde transport within 60-90 min (Fig. 3D; T90: $59.35 \pm 12.15 \%$, $\left.F_{(5,25)}=8.796, p=0.0014, n=6\right)$ and anterograde transport within 90-120 $\mathrm{min}$ (Fig. 3D; T120: $61.4 \pm 6.3 \%, F_{(5,25)}=7.302$, $p=0.0006, n=6)$.

Under baseline conditions (T0), the mean distance of mitochondrial moving events measured was $13.7 \pm 0.6 \mu \mathrm{m}$, with similar distances traveled in both directions (antero: $13.8 \pm 8.1 \mu \mathrm{m}$ vs retro: $12.7 \pm 6.2 \mu \mathrm{m} ; t_{(98)}=1.013, p=0.3135$, two-tailed unpaired $t$ test, $n=50$, data not shown). Treatment of the axonal compartment with $0.1 \mathrm{~mm}$ AICAR significantly reduced the traveling distance of mitochondrial movements, occurring again notably earlier in the retrograde (Fig. $3 E$; T60: $53.1 \pm 9.4 \%, F_{(5,25)}=$ 9.358, $p=0.0011, n=6)$ than the anterograde direction (T120: $\left.56.2 \pm 10.5 \%, F_{(5,25)}=4.245, p=0.0061, n=6\right)$. At the end stage of the experiment, the mean distance of retrograde and anterograde movements was $35.5 \pm 9.0 \%$ and $58.5 \pm 15.0 \%$ of their respective baseline values.

Coapplication with the AMPK antagonist, CC $(10 \mu \mathrm{M})$, successfully reversed AICAR-mediated effects in all parameters measured, suggesting the direct involvement of AMPK signaling in the acute modulation of local mitochondrial transport. Interestingly, treatment of the axonal compartment with CC alone did not affect the mean frequency, velocity, or distance of the mitochondrial movements in either direction, suggesting that acute inhibition of physiological AMPK signaling does not modulate mitochondrial transport kinetics. Local AICAR treatment at the distal axon also caused a mild, yet nonsignificant, trend toward an increase in the proportion of stationary mitochondria over time, reaching $120 \pm 25 \%$ of baseline values by the end of the experiment, whereas coapplication with CC maintained the proportion of stationary mitochondria at $104 \pm 11 \%$ of baseline values (data not shown).

Together, these results suggest that acute activation of AMPK at the distal axon dampens the kinetics of mitochondrial transport mechanisms within the local axon segment, with a significant influence in particular on retrograde transport.

Local AMPK activation at the distal axon induces direct accumulation of mitochondria to this region

Retrograde transport of mobile mitochondria was found to be more sensitive to AICAR treatment, as transport in this direction was affected earlier than anterograde transport for all parameters analyzed. Thus, to investigate whether the differential sensitivities of bidirectional mitochondrial movements influence overall mitochondrial distribution, we focused our attention on mitochondrial density at the distal axon directly exposed to AICAR treatment. We performed time lapse confocal imaging within the axonal compartment of the microfluidic device before (T0) and 2 h (T120) after AICAR addition to this compartment (Fig. 4A). The number of GFP-tagged mitochondria in the axon termini was significantly increased $2 \mathrm{~h}$ following AICAR treatment at this region (Fig. $4 B I I I ; 109.8 \pm 1.8 \%$ of baseline, $t_{(31)}=3.96$, $p=0.0004$, two-tailed paired $t$ test, $n=32$ FOVs from 6 experiments). Cotreatment with CC (Fig. 4BIV) antagonized this effect, confirming an AMPK-mediated effect. Acute inhibition of AMPK with CC alone did not significantly alter mitochondrial distribution in this region (Fig. $4 B V ; 98.7 \pm 1.7 \%$ of baseline, $t_{(45)}=1.545 p=0.4833$, two-tailed paired $t$ test, $n=35$ FOVs from 6 experiments). These data suggested that the differential sensitivity of anterograde and retrograde mitochondrial transport to AICAR treatment promoted accumulation of mitochondria at the site of enhanced AMPK signaling.

We finally explored whether AICAR treatment at the distal axon promoted the accumulation of energized mitochondria at this site. The maintenance of the mitochondrial membrane potential is critical for bioenergetic function of the mitochondria. TMRM, a fluorescent probe sensitive to changes in mitochondrial membrane potential, was used as an indicator of mitochondrial bioenergetic status. Interestingly, we observed that the membrane potential of mitochondria treated with AICAR was significantly reduced, as indicated by a reduction in TMRM fluorescence of distal mitochondria after $2 \mathrm{~h}$ treatment (Fig. 4CI; $83.9 \pm 2.8 \%$ of baseline values, $F_{(3,154)}=3.111, p=0.0177$ compared with control, $n=38$ FOVs from 9 biological replicates), which may be indicative of increased mitochondrial workload. AICAR-induced changes in TMRM fluorescence were independent of the plasma membrane potential as no alterations in $\mathrm{DiSBAC}_{4}(3)$ fluorescence were observed (Fig. 4CIII; control: $98.3 \pm 0.7 \%$, AICAR: $98.1 \pm 1.0 \%$ of baseline values, $t_{(140)}=$ $0.2072, p=0.8362$, two-tailed unpaired $t$ test, $n=69$ FOVs from four biological replicates).

Cotreatment of AICAR with CC removed this effect, maintaining TMRM fluorescence signal at levels similar to control values (Fig. 4CII; control: $93.6 \pm 1.1 \%$, AICAR+CC: $91.2 \pm 2.3 \%$ of baseline values, $\left.F_{(3,154)}=3.111, p=0.8177, n=6\right)$. As a positive control, application of $10 \mu \mathrm{M}$ FCCP, which potently uncouples mitochondrial oxidative phosphorylation processes, resulted in a depletion of TMRM fluorescence to $36.7 \pm 7.8 \%$ of pretreatment values. Together, these results suggest that mild activation of AMPK signaling at the distal compartment induces accumulation of mitochondria to this region and depolarization of their mitochondrial membrane potential in response to this energetic stress stimulus. We have previously reported that AMPK activation induces an increase in ATP levels (Anilkumar et al., 2013), and the mitochondrial membrane depolarization seen in this study may therefore be induced by increased mitochondrial workload.

\section{Substitution of glucose with lactate as the primary energy} source at the distal axon alters local mitochondrial transport Axonal mitochondria have been shown to preferably use lactate as their energy source, with lactate alone being sufficient to maintain axonal function and plasticity (Pellerin et al., 1998; Magistretti and Allaman, 2018). To study whether these findings 
A

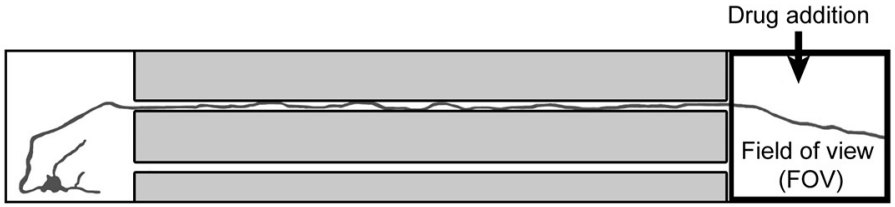

All

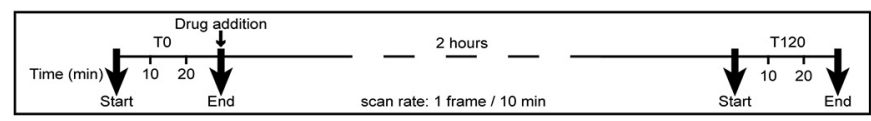

BI

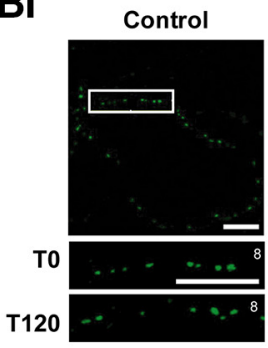

AICAR + CC

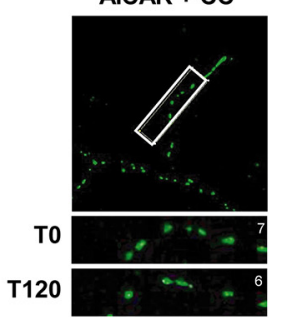

CI
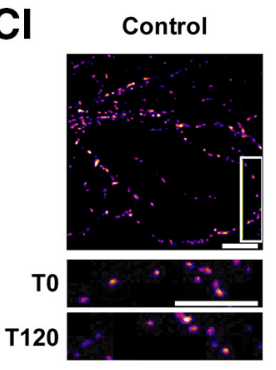

$A I C A R+C C$
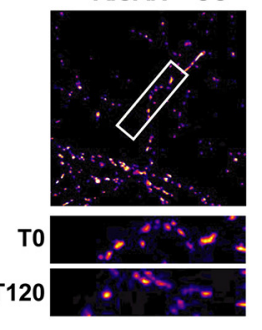

AICAR

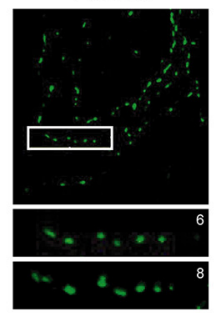

CC

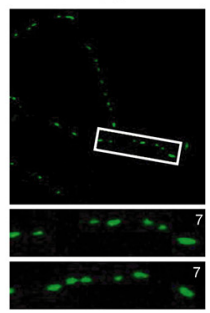

AICAR
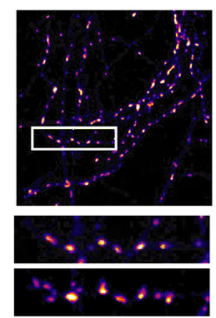

CC

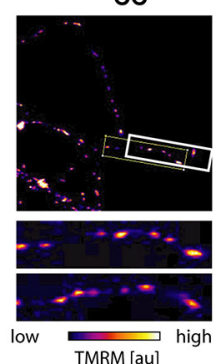

BII

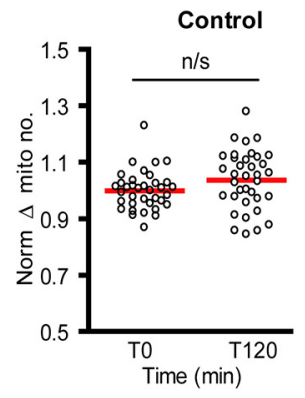

BIV

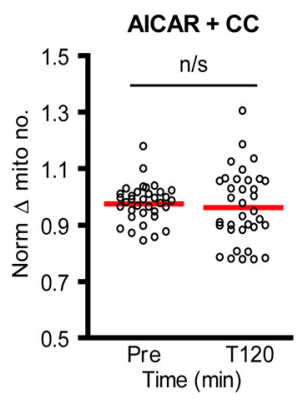

\section{BIII}

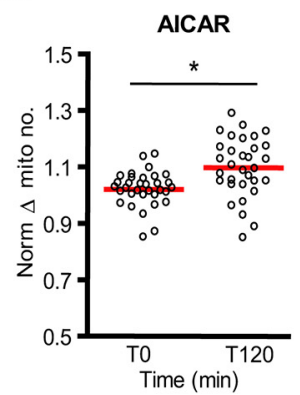

BV

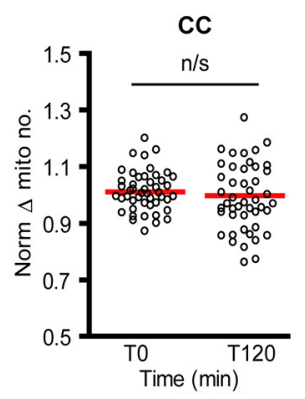

CII

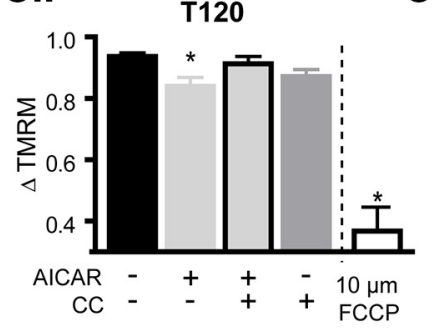

C III

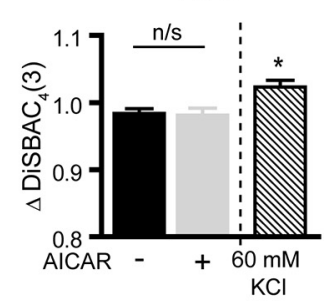

Figure 4. Localized AMPK activation at the distal axon induced an accumulation of mitochondria directly at the site of treatment. $A$, Schematic of experiment design. Fluorescent confocal images were captured at the distal axon (FOV) for $30 \mathrm{~min}$ at a rate of 1 frame/10 min, $30 \mathrm{~min}$ before (TO) and $2 \mathrm{~h}$ after (T120) treatment with $0.1 \mathrm{~mm}$ AICAR $\pm 10 \mu \mathrm{m}$ CC directly to this region. $B$ I, Representative images $(354.2 \mu \mathrm{m} \times 354.2 \mu \mathrm{m})$ of mito-GFP-positive mitochondria within the axonal compartment directly exposed to treatment. Section of distal axon (white box) expanded below to show the number of mitochondria before (TO) and $2 \mathrm{~h}$ after treatment (T120). Mitochondrial counts for the section noted in the top right-hand corner of each image. Scale bars, $20 \mu \mathrm{m}$. BII-BV, Quantification of the mean number of mitochondria in the distal axon before and $2 \mathrm{~h}$ after AICAR $\pm C($ treatment at this region. AICAR treatment (BIII) at the distal axon resulted in an accumulation of mitochondria in this region after $2 \mathrm{~h}$, an effect removed by cotreatment with $C\left(\right.$ (BIV). ${ }^{*} p \leq 0.001$ (two-tailed paired $t$ test). $n \geq 32 \mathrm{FOV}$ s from at least four independent culture preparations. C, Cortical neurons were preloaded with $20 \mathrm{~nm}$ TMRM for 30 min before imaging. Cl, Representative images of mitochondrial TMRM fluorescence levels within the axonal compartment at the start of the experiment. Section of the distal axons within the white box is expanded below to show TMRM fluorescence before (TO) and $2 \mathrm{~h}$ after treatment (T120). Scale bars, $20 \mu \mathrm{m}$. CII, AICAR treatment resulted in a mild decrease in mitochondrial membrane potential, compared with control. FCCP, a potent uncoupler of oxidative phosphorylation, was used as a positive control. ${ }^{*} p \leq 0.05$ compared with control levels at T150 (one-way ANOVA with Dunnett's multiple-comparison post hoc test). $n \geq 32$ FOVs from at least four independent culture preparations. FCCP data were obtained from 23 FOVs from two experiments. CIII, Cells were preloaded with $1 \mu \mathrm{m}$ DiSBAC $(3)$ for 30 min before imaging, to visualize changes in the plasma membrane potential. The $2 \mathrm{~h}$ AICAR treatment did not alter the plasma membrane potential. Two-tailed unpaired $t$ test. $n=69 \mathrm{FOV}$ from at least four independent culture preparations. Potassium chloride $(\mathrm{KCl}, 60 \mathrm{~mm})$ was used as a positive control for depolarization of the plasma membrane, resulting in a significant increase in DiSBAC $(3)$ fluorescence. $\mathrm{KCl}$ data were obtained from 40 FOVs from four experiments. ${ }^{*} p \leq 0.05$ compared with control levels at T150. 
Al

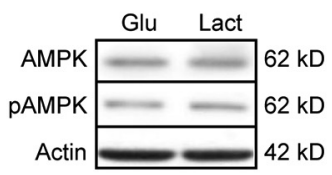

II

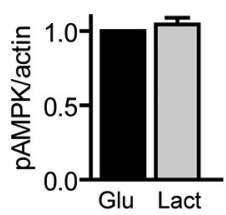

III

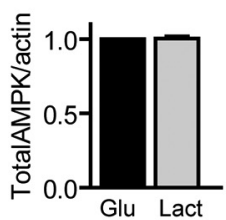

IV

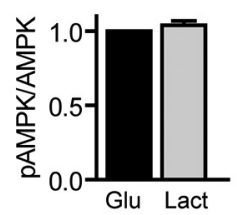

B

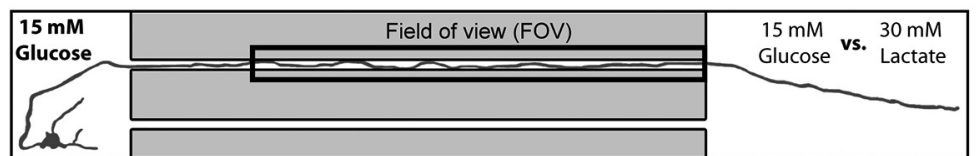

C

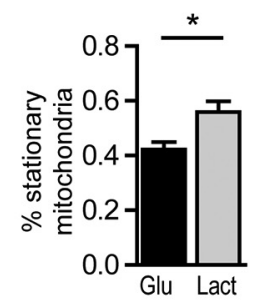

$15 \mathrm{mM}$ Glucose (Glu) $30 \mathrm{mM}$ Lactate (Lact)
D

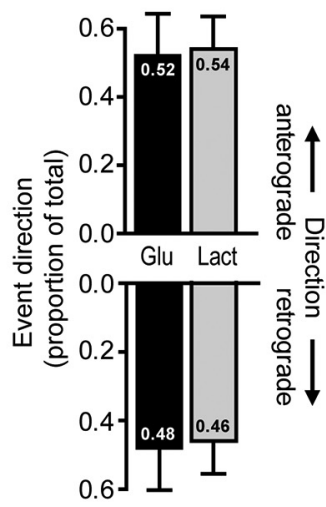

E

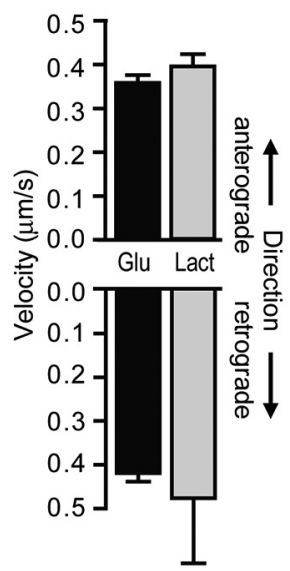

F

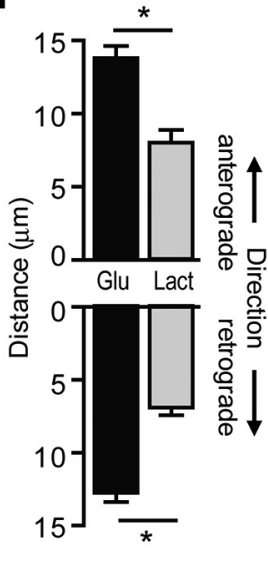

Figure 5. Localized substitution of glucose for lactate at the distal axon reduced mean distance of mitochondrial transport events in both directions. $\boldsymbol{A}$, Representative Western blot image and quantification of group data confirm that neither (AI) phosphorylated AMPK (pAMPK) nor (AIII) total AMPK levels are altered when glucose is substituted for the metabolic equivalent concentration of lactate as the cell's main energy source. AIV, The ratio of pAMPK to total AMPK is also unchanged. Beta-actin serves as a loading control. $p>0.05$ (two-tailed unpaired $t$ test). Western blots were prepared from three independent culture preparations. $\boldsymbol{B}$, Schematic of experimental design. The somatodendritic compartment was exposed to glucose-rich buffer while the axonal compartment was exposed either to buffer containing glucose $(15 \mathrm{~mm})$ or the metabolic equivalent concentration of lactate ( $30 \mathrm{~mm})$. Baseline mitochondrial mobility was measured in the distal axon segment contained in the microgroove (FOV) for $30 \mathrm{~min}$. The kinetics of mitochondrial transport events were compared in axons, which were exposed locally to glucose (Glu) or lactate (Lact) as their main energy source. C, Axons exposed to lactate showed a significantly higher proportion of stationary mitochondria than those exposed to glucose. D, Substitution of glucose with lactate did not affect the proportion of mitochondrial transport events or the velocity $(\boldsymbol{E})$ in either direction. However, a significant change in the mean distance $(\boldsymbol{F})$ of mitochondrial movement events in both anterograde and retrograde directions was noted. ${ }^{*} p<0.0001$ (two-tailed unpaired $t$ test). $n \geq 21$ from at least five independent culture preparations.

could also extend to mitochondrial transport dynamics, we substituted glucose with the metabolic equivalent concentration of lactate in the axonal compartment and monitored baseline (30 min) mito-GFP transport in the adjacent axon segment, as before (Fig. $5 B$ ). First, we noted by Western blotting that total AMPK and phosphorylated AMPK levels remained at similar levels in neurons exposed to glucose or its metabolic equivalent concentration of lactate, suggesting that energetic homeostasis was maintained with this change in energy substrate (Fig. 5A).

Analysis of axonal mitochondria over 30 min baseline recording revealed a higher percentage of stationary mitochondria within the axons locally exposed to lactate (Fig. $5 C$; glucose: $42.2 \pm 2.7 \%, n=33$, lactate: $55.9 \pm 3.9 \%, n=22, t_{(53)}=2.951$, $p=0.0047$, two-tailed unpaired $t$ test). Focusing on the mobile mitochondria, we found no directional change in the proportion of anterograde and retrograde mitochondrial movement events in axons exposed to glucose or lactate in the axonal compartment (Fig. 5D). We also found that the change in energy substrate at the distal axon did not alter the mean velocity of these movement events in either direction (Fig. 5E). Interestingly, substitution of glucose with lactate at the axonal compartment did induce over a $50 \%$ decrease in the mean distance of traveled in both anterograde and retrograde directions of mobile mitochondria in the adjacent axon (Fig. 5F; anterograde: Glu: $13.81 \pm 0.81$ $\mu \mathrm{m}, n=50$ vs Lact: $8.01 \pm 0.86 \mu \mathrm{m}, n=21, t_{(69)}=4.208, p<$
0.0001 ; retrograde: Glu: $12.77 \pm 0.62 \mu \mathrm{m}, n=50$ vs Lact: 6.91 $\pm 0.52 \mu \mathrm{m}, n=21 ; t_{(69)}=5.717, p<0.0001$, two-tailed unpaired $t$ tests). Together, these data suggest that, although axonal lactate has been reported to be sufficient to maintain axonal function and plasticity, it may induce subtle effects on mitochondrial transport kinetics compared with the metabolic equivalent concentration of glucose.

Local nutrient deprivation at the distal axon reduces the frequency, velocity, and distance of axonal mitochondrial movement in an AMPK-dependent manner

In order to confirm a role for AMPK-mediated modulation of local mitochondrial transport in a more physiological setting, we next established an in vitro microfluidic model of localized nutrient deprivation at the distal axon to mimic white matter energetic stress. As lactate is taken up into neurons via the monocarboxylate transporter 1 and monocarboxylate transporter 2, we first tested at a whole-cell level whether we could mimic cellular energy deficiency and activate AMPK signaling by pharmacological antagonism of these transporters using AR-C155858. Western blotting confirmed that AR-C155858 (10 nM) sufficiently inhibited lactate uptake to evoke a rapid phosphorylation of AMPK (Fig. 6AII; T30: 217.1 $\pm 24.7 \%$ of baseline values, $\left.F_{(4,15)}=3.078, p=0.0360, n=4\right)$, verifying the activation of this 

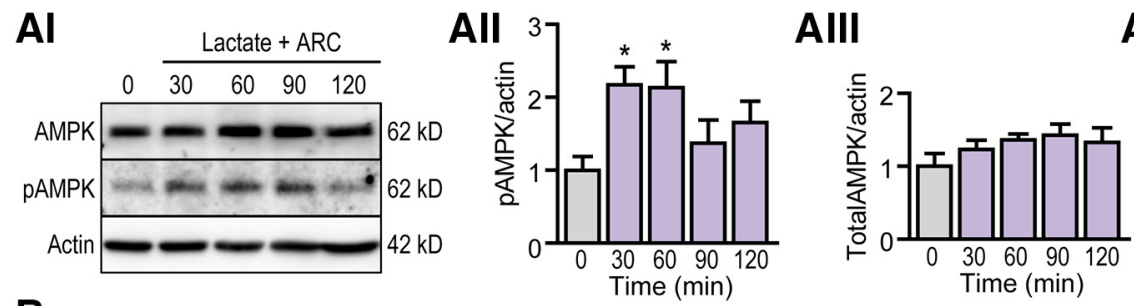

AIV

B

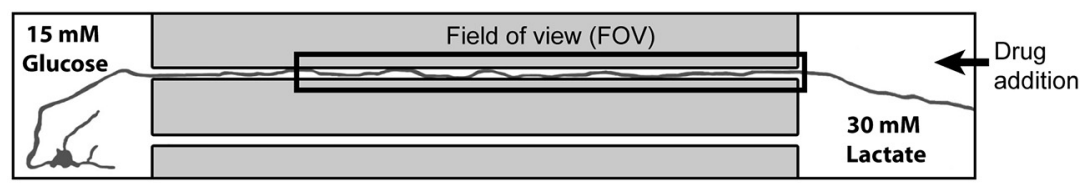

Cl

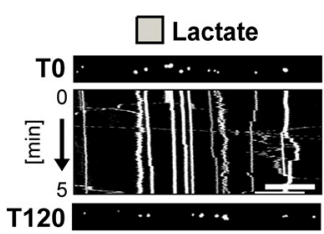

D

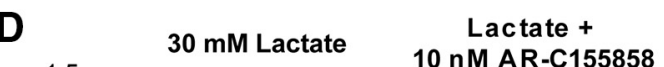

Lactate +
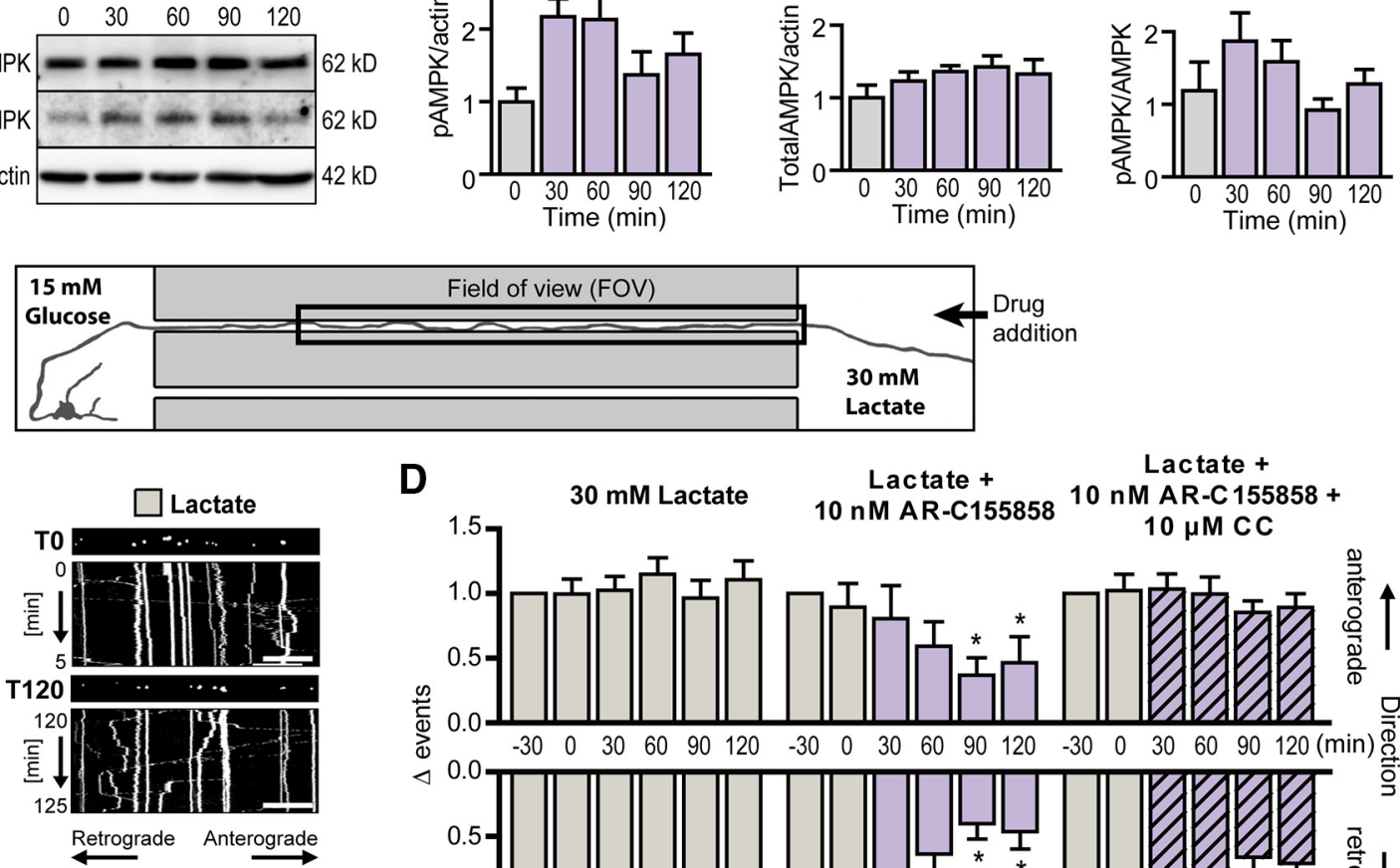

CII

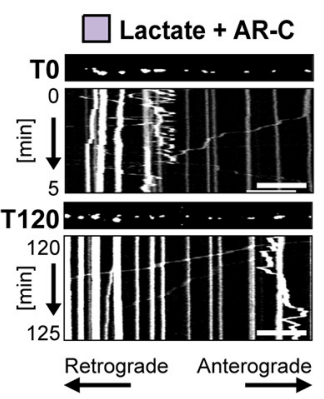

CIII
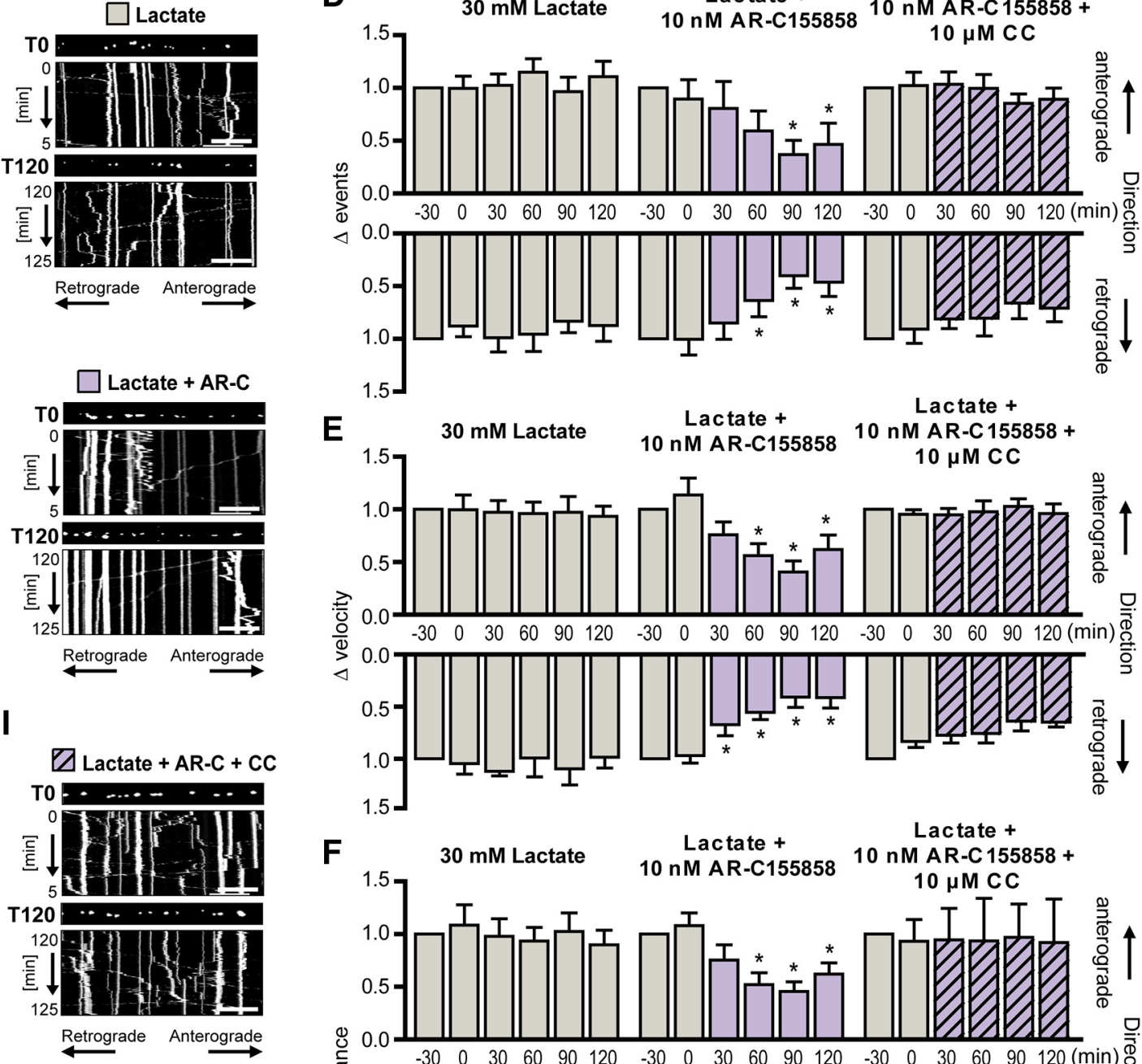

Lactate +
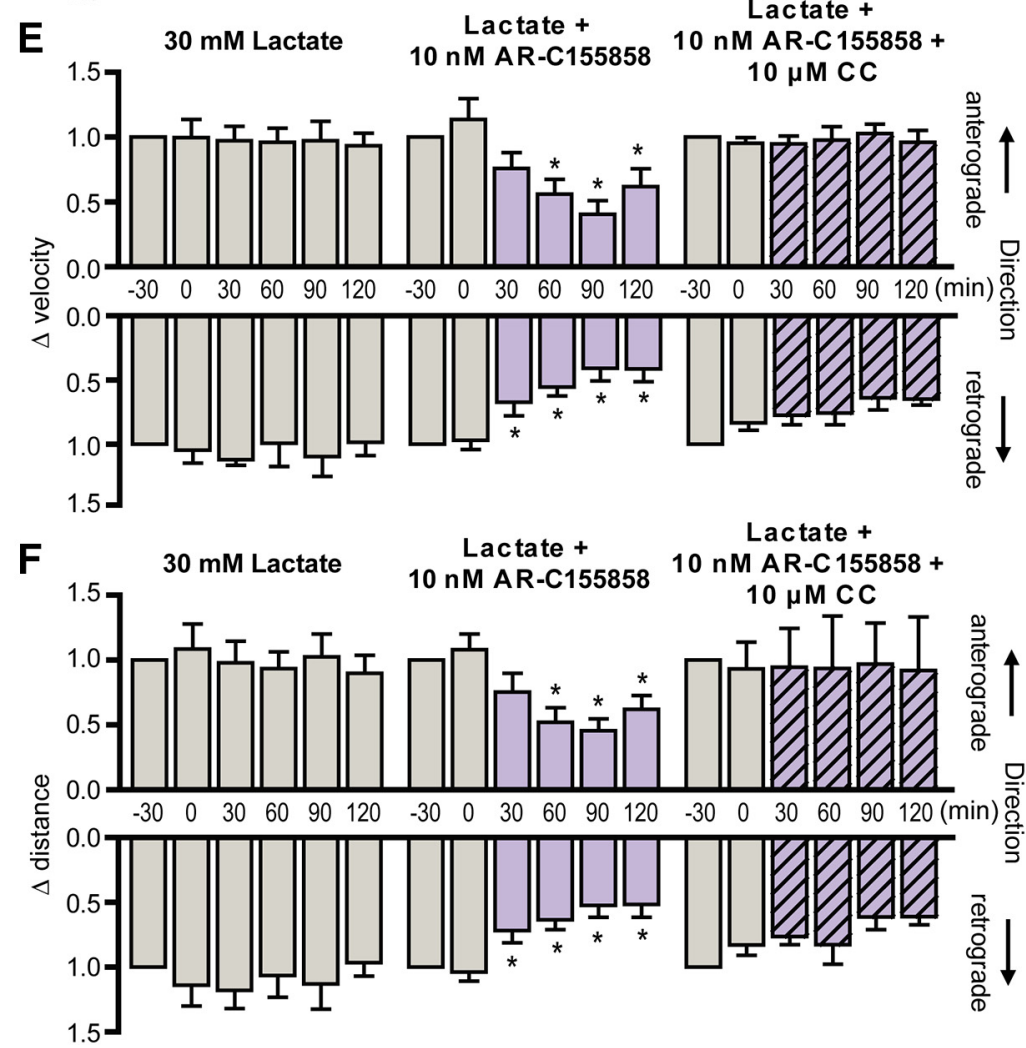

Figure 6. Establishment of an in vitro microfluidic model of localized nutrient deprivation. $\mathbf{A l}$, Representative Western blot image showing total cellular AMPK levels and its phosphorylation status during localized nutrient deprivation (Lactate + ARC). Beta-actin served as a loading control. All, Quantification of phosphorylated AMPK (pAMPK) relative to $\beta$-actin levels showed a marked increase in PAMPK in response to inhibition of lactate uptake with AR-C188585 within $30 \mathrm{~min}$. AlII, Quantification of total AMPK relative to $\beta$-actin. AIV, Quantification of pAMPK relative to total AMPK. ${ }^{*} p<0.05$ (one-way ANOVA with Dunnett's multiple-comparison post hoc test). $n=4$ from four independent culture preparations. $\boldsymbol{B}$, Schematic of experimental design. The somatodendritic compartment was exposed to glucose-rich $(15 \mathrm{~mm})$ experimental buffer while glucose was substituted with the metabolic equivalent concentration of lactate ( 30 mm) at the axonal compartment. Mitochondrial mobility was measured in the axon segment contained in the microgroove (FOV) adjacent to the area of drug addition (axonal compartment). The experimental time course illustrated in Figure 2All was used, with drug addition to the axonal compartment after 60 min baseline recording. Statistical analysis was performed by comparing each time interval to $30 \mathrm{~min}$ baseline recording before drug addition (TO). C, Representative kymographs of mitochondrial mobility in the axon segment (FOV) during baseline recording (T0) and $2 \mathrm{~h}$ 
energetic stress sensor in response to diminished energy substrate availability.

This concentration of AR-C155858 was consequently chosen to induce nutrient deprivation in our in vitro microfluidic model of localized axonal nutrient deprivation. As before, mito-GFPpositive mitochondria were monitored in the axon segment adjacent to the axonal compartment by time-lapse confocal imaging (marked as FOV in Fig. 6B). The axonal compartment was exposed to experimental buffer enriched with $30 \mathrm{~mm}$ lactate as their energy source. After $60 \mathrm{~min}$ of baseline recording, $10 \mathrm{~nm}$ AR-C155858 was added to the lactate-enriched buffer in the axonal compartment to inhibit local lactate uptake. Confocal images were taken for a further $2 \mathrm{~h}$ to monitor temporal changes in $\mathrm{mi}$ tochondrial transport induced by inhibition of axonal lactate uptake. The time series of images were then subdivided into kymographs to graphically represent mitochondrial movement for each $30 \mathrm{~min}$ time interval (Fig. 6C). Data were then extracted from each kymograph and analyzed as described previously. Interestingly, we noted that the mean frequency, velocity, and distance of mitochondrial transport events in axons which were exposed to lactate remained stable for the duration of the experiment, suggesting an adaptation of the axons to lactate as their substitute energy source (Fig. $6 D-F$ ).

AR-C155858 treatment at the distal axon induced an increase in the proportion of stationary mitochondria over time, reaching $141 \pm 13 \%$ of baseline values $\left(\mathrm{T} 120, F_{(4,24)}=5.254, p=0.0034\right.$, $n=7$; data not shown) by the end of the experiment, whereas coapplication with CC maintained the proportion of stationary mitochondria at $113 \pm 13 \%$ of baseline values $\left(\mathrm{T} 120, F_{(4,24)}=\right.$ $0.5359, p=0.4990, n=7$; data not shown). Focusing on the transport dynamics of the mobile mitochondria in the axon segment, we observed a marked decline in the frequency of mitochondrial transport events in both anterograde and retrograde directions in response to local nutrient deprivation at the distal axon, reaching statistical significance at $90 \mathrm{~min}$ and $60 \mathrm{~min}$ post-treatment, respectively (Fig. $6 D$; antero: T90: $36.9 \pm 13.2 \%, F_{(4,24)}=6.295$, $p=0.0021, n=7$; retro: T60: $63.79 \pm 15.4 \%, F_{(4,24)}=15.25$, $p=0.003, n=7)$. Addition of AR-C155858 to the lactate-rich buffer in the axonal compartment also resulted in a significant depression in the mean velocity of mitochondrial transport, an effect that may be attributed primarily to the rapid, strong deceleration in retrograde mitochondrial movements (Fig. 6E; Lactate + AR-C155858; T30: $67.37 \pm 10.6 \%$ of baseline, $F_{(4,24)}=16.25$, $p=0.0069, n=7)$ with a more delayed, milder effect on

\section{$\leftarrow$}

(T120) after treatment of the distal axon with (CI) $30 \mathrm{~mm}$ lactate, (CII) lactate and $10 \mathrm{~nm}$ ARC1557858, or (CIII) lactate, AR-C155858 and $10 \mu \mathrm{m}$ CC. Mito-GFP-tagged mitochondria can be visualized in the still image shown above each kymograph (first image of corresponding time period). Scale bar, $20 \mu \mathrm{m}$. $\boldsymbol{D}$, Inhibition of lactate uptake into the distal axon by addition of AR-C155858 to this region induced a depression in the frequency of retrograde mitochondrial movements (T90) with a milder depression in the frequency of anterograde movement events. This effect was reversed by cotreatment with $\mathrm{CC}$ confirming a role for AMPK signaling in nutrient deprivation-induced depression in local mitochondrial transport frequency. $\boldsymbol{E}, \boldsymbol{F}$, The mean velocity and distance of mitochondrial movement events were first normalized to their respective pretreatment values (T30). $\boldsymbol{E}$, The velocity of bidirectional mitochondrial transport remained stable for the duration of the experiment in axons exposed distally to lactate as their energy source. AR-C155858 induced a maintained depression of retrograde mitochondrial transport velocity within $30 \mathrm{~min}$ (T30), with a milder effect evident in anterograde transport at a later time point (T90). F, AR-C155858 also rapidly reduced the mean distance of retrograde mitochondrial transport (T30) with the mean distance of anterograde transport affected at a later time point (T60). ${ }^{*} p \leq 0.01$ (repeated-measures ANOVA with Dunnett's multiple-comparison post hoc test). $n=7$ experiments from at least five independent culture preparations. anterograde mitochondrial movements (T60). Similarly, ARC155858 induced a sustained depression in the mean distance of mitochondrial movement events in both directions, with the effect more pronounced in the retrograde direction (Fig. 6F; Lactate + AR-C155858, T30: $71.93 \pm 9.3 \%$ of baseline, $F_{(4,24)}=$ 14.28, $p=0.0026, n=7)$. Antagonism of AMPK activity with coapplication of CC reversed the effects of localized nutrient deprivation on all parameters measured (Fig. 6D-F; Lactate + ARC155858 + CC). Together, these results suggest that localized nutrient deprivation at the distal axon induces a depression in axonal mitochondrial transport through local activation of the energetic stress sensor, AMPK.

\section{Discussion}

The physiological role of AMPK in the regulation of cellular energy homeostasis has been well established, while the underlying mechanisms of the multiple downstream signaling pathways involved are yet to be fully elucidated. AMPK signaling promotes local ATP generation and/or restrains biosynthetic pathways, with the common goal to enhance ATP availability for critical homeostatic mechanisms while temporarily minimizing ATP consumption during the period of energetic stress (for review, see Hardie et al., 2012; Herzig and Shaw, 2018). As mitochondria are the primary producers of energy within the neuron, their continual, bidirectional mobility plays a pivotal role in the capacity of the neuron to tolerate local, temporal energetic disturbances. Here, we demonstrate that AMPK signaling also plays a role in the modulation of mitochondrial transport by locally suppressing their mobility during periods of energetic stress.

Mitochondria are trafficked throughout the axon by ATPase motor proteins, along a uniformly organized microtubule network (Schroer et al., 1988; Schnapp and Reese, 1989; Pilling et al., 2006). Mitochondria themselves therefore rely on ATP for their own transport. Indeed, previous studies indicate that the loss of mitochondrial ATP production without disruption of its membrane potential or morphology may have a negative effect on their own mobility (Rintoul et al., 2003; Kaasik et al., 2007; Zala et al., 2013). Developmental studies have suggested a relationship between AMPK signaling and mitochondrial mobility in immature neurons. Genetic knockdown of LKB1, an upstream activator of AMPK, or NUAK1 (AMPK-related protein kinase 5 ), enhanced the proportion of mobile mitochondria along these axons, whereas overexpression of these kinases had the opposite effect (Courchet et al., 2013). Using the neuroprotective paradigm of pharmacological AMPK activation previously established in our laboratory (Anilkumar et al., 2013), we here explored the effect of acute localized AMPK activation on axonal mitochondrial transport.

AICAR-mediated AMPK activation localized to the axonal compartment suppressed mitochondrial mobility in the adjacent axon segment by reducing the mean frequency, velocity, and distance of their movement events. A similar effect was seen in our in vitro microfluidic model of localized nutrient deprivation, whereby the axons were deprived of their energy substrate through inhibition of lactate uptake transporters, inducing energetic stress and subsequent activation of AMPK. In both experimental paradigms, pharmacological antagonism of AMPK with CC prevented the depression in mitochondrial mobility in all parameters measured, confirming the role of this energetic stress sensor in local modulation of mitochondrial transport. As mitochondrial mobility is governed by consumption of energy by ATPase motor proteins, depression of their transport may 
contribute to AMPK-mediated energy conservation, allowing ATP to be redirected to more critical energy-consuming processes during periods of energetic stress.

Local AMPK activation also led to a decrease in TMRM fluorescence, indicative of mild mitochondrial depolarization which is likely explained by an increased mitochondrial workload. We previously reported that AICAR-induced AMPK activation increases neuronal ATP levels within $2 \mathrm{~h}$ (Anilkumar et al., 2013). However, isolated AICAR treatment at the distal axon in our system did not induce detectable changes in ATP levels in this region (data not shown), although the low volume of axonal mitochondria relative to the soma may account for the lack of detectable changes of axoplasmic ATP levels in this experimental setup. However, it is plausible that ATP levels are indeed maintained at a very constant level in axons, or that any increase in ATP production is coupled with enhanced ATP hydrolysis during AMPK activation. Mitochondrial depolarization may also be indicative of increased proton leak or decreased respiratory complex activity (Zorova et al., 2018).

We also report an enhanced sensitivity of retrograde transport to both AICAR treatment and localized nutrient deprivation at the distal axon in all parameters studied. Under baseline conditions, mitochondrial transport was found to be more rapid in the retrograde than anterograde direction (data not shown). This observation reflects the differential kinetics between the principal transport motor proteins, with kinesin-mediated anterograde transport occurring in $8 \mathrm{~nm}$ steps and dynein-mediated retrograde transport in steps varying from 8 to $24 \mathrm{~nm}$ (Coy et al., 1999; Reck-Peterson et al., 2006). The enhanced sensitivity of retrograde mitochondrial transport to AMPK signaling may act as a neuroprotective mechanism by delaying mitochondrial movement away from the site of enhanced AMPK activity in an effort to retain mitochondria in the region of enhanced energetic stress. Indeed, analysis of the distal axon revealed that AICAR induced a marked accumulation of mitochondria to this region, along with a mild depolarization of the mean mitochondrial membrane potential in this region. This was not observed, however, in experiments of localized axonal nutrient deprivation, perhaps due to differences in the activation profiles of local AMPK signaling between these two experimental paradigms.

One attractive hypothesis that may contribute to the effects of AMPK signaling on local mitochondrial transport is the potential for AMPK to influence its microenvironment. Previous work in our laboratory identified enhanced surface expression of the GLUT3 glucose transporter within $2 \mathrm{~h}$ of exposure to AICAR (Weisova et al., 2009; Anilkumar et al., 2013). Upregulation of GLUT3 in response to AMPK signaling facilitates glucose uptake into the cell, providing extra substrate for mitochondrial ATP synthesis during periods of energetic stress. Indeed, elevated cytoplasmic glucose has been shown to promote O-GlcNAc transferase-mediated GlcNAcylation of Milton, reducing its ability to recruit kinesins to mitochondria, leading to a reduction in mitochondrial mobility in both directions (Glater et al., 2006; Pekkurnaz et al., 2014). It has also been reported that local elevation of glucose at the distal axon, and subsequent spatial inhomogeneity of axoplasmic glucose, results in mitochondrial accumulation at this region (Pekkurnaz et al., 2014). Thus, AMPK-mediated GLUT3 upregulation and intracellular glucose accumulation may account for the recruitment of mitochondria to this region seen with AICAR treatment at the distal axon. As our in vitro model of localized nutrient deprivation is void of glucose in the axon compartment, activation of AMPK in this paradigm cannot induce glucose-mediated accumulation of mitochondria to this region, which may explain why this was not observed in these experiments.

AMPK-mediated depression of local mitochondrial mobility may also be attributed to numerous underlying signaling cascades targeting any of the functional connections between the cytoskeletal, motor, adaptor, and mitochondrial proteins. The neuronal cytoskeletal proteins actin, tubulin, and neurofilament have all been identified as potential AMPK substrates (Tuerk et al., 2007). These proteins not only provide structural support to the neuron but provide a platform for the transport of organelles, including mitochondria, along neuronal processes, and assist mitochondrial docking (Kuznetsov et al., 1992; Morris and Hollenbeck, 1995; Uchida et al., 2009). Pharmacological disruption of microtubules in mature neurons has been reported to reduce the velocity and distance of mitochondrial transport, whereas disruption of actin filaments reduced the frequency of mitochondrial movement in these cells (Morris and Hollenbeck, 1995). The microtubule-associated protein Tau facilitates motor protein engagement with microtubules during long-distance organelle transport. AMPK has been shown to directly phosphorylate Tau protein, altering its binding properties to motor proteins, which may slow down and impair organelle transport (LlorensMartín et al., 2011; Thornton et al., 2011). Indeed, abnormal accumulation of phospho-AMPK and concomitant hyperphosphorylated Tau protein have been reported in cerebral neurons in tauopathies, such as Alzheimer's disease, impairing mitochondrial transport in these cells (Reddy, 2011). Putative AMPK phosphorylation sites are also found on many motor proteins involved directly in mitochondrial transport, such as members of the kinesin superfamily, KLC1, KLC2, and KIF5B, the myosin light chain 2, and dynein intermediate chain 2 (Tuerk et al., 2007; Schaffer et al., 2015). However, this may not necessarily infer AMPK-mediated alteration of their motor activity, as a study conducted in pancreatic $\beta$ cells found that AMPK phosphorylation of KLC1 and KIF5B did not influence their motor function with respect to insulin granule transport (McDonald et al., 2009). There are also other phosphorylation targets that may be affected by AMPK activation or nutrient/energy deprivation which require further interrogation.

Interestingly, isolation of AICAR treatment to the somatodendritic region of the neurons did not influence mitochondrial transport kinetics within the spatially isolated axon segment, suggesting regional-specific effects of local AMPK activation. It is widely documented that a large pool of mitochondria are found in the soma (also observed in this study; Fig. 1A) to meet the constant high-energy demands of the nucleus and organelles in this region. It is possible, that in the case of AMPK stimulation in this region, the pool of somatic mitochondria is sufficient to respond to the local increased energy stress signal, allowing the axonal mitochondrial population to continue to distribute and supply energy within the axon. The density of cells in the somatodendritic compartment is also much greater than that of the select few axons that project into the axonal compartment; thus, a substantial difference between the total numbers of mitochondria within these two compartments may also contribute to the region-specific responses to AMPK activation.

This study highlights the capacity of spatially confined AMPK signaling to influence the transport dynamics of the local pool of axonal mitochondria. Direct (AICAR treatment) and indirect (nutrient deprivation) activation of AMPK represses the frequency and kinetics of localized mitochondrial movements, potentially minimizing energy consumption by motor proteins in this region and enhancing energy availability for more critical 
processes. This effect is region-specific, as AMPK activation in the soma failed to influence mitochondrial transport within the spatially isolated distal axon. These findings suggest that axons may have the local capability to sense and combat axonal energetic disturbances by modulating local mitochondrial transport, an effect mediated by the energetic stress signaling molecule AMPK. This novel role for AMPK may further contribute to neuronal preconditioning by enhancing the cell's local capacity to restore energetic homeostasis in this region if further perturbation occurs.

\section{References}

Anilkumar U, Weisova P, Dussmann H, Concannon CG, Konig HG, Prehn JH (2013) AMP-activated protein kinase (AMPK)-induced preconditioning in primary cortical neurons involves activation of MCL-1. J Neurochem 124:721-734.

Blumbergs PC, Scott G, Manavis J, Wainwright H, Simpson DA, McLean AJ (1995) Topography of axonal injury as defined by amyloid precursor protein and the sector scoring method in mild and severe closed head injury. J Neurotrauma 12:565-572.

Chabriat H, Pappata S, Poupon C, Clark CA, Vahedi K, Poupon F, Mangin JF, Pachot-Clouard M, Jobert A, Le Bihan D, Bousser MG (1999) Clinical severity in CADASIL related to ultrastructural damage in white matter: in vivo study with diffusion tensor MRI. Stroke; a journal of cerebral circulation 30:2637-2643.

Chada SR, Hollenbeck PJ (2004) Nerve growth factor signaling regulates motility and docking of axonal mitochondria. Curr Biol 14:1272-1276.

Chambers TW, Daly TP, Hockley A, Brown AM (2014) Contribution of glycogen in supporting axon conduction in the peripheral and central nervous systems: the role of lactate. Front Neurosci 8:378.

Chang DT, Honick AS, Reynolds IJ (2006) Mitochondrial trafficking to synapses in cultured primary cortical neurons. J Neurosci 26:7035-7045.

Chen Y, Sheng ZH (2013) Kinesin-1-syntaphilin coupling mediates activitydependent regulation of axonal mitochondrial transport. J Cell Biol 202:351-364.

Cheng A, Hou Y, Mattson MP (2010) Mitochondria and neuroplasticity. ASN neuro 2:e00045.

Concannon CG, Tuffy LP, Weisova P, Bonner HP, Davila D, Bonner C, Devocelle MC, Strasser A, Ward MW, Prehn JH (2010) AMP kinasemediated activation of the $\mathrm{BH} 3$-only protein Bim couples energy depletion to stress-induced apoptosis. J Cell Biol 189:83-94.

Courchet J, Lewis TL, Jr., Lee S, Courchet V, Liou DY, Aizawa S, Polleux F (2013) Terminal axon branching is regulated by the LKB1-NUAK1 kinase pathway via presynaptic mitochondrial capture. Cell 153:1510-1525.

Coy DL, Wagenbach M, Howard J (1999) Kinesin takes one 8-nm step for each ATP that it hydrolyzes. J Biol Chem 274:3667-3671.

Culmsee C, Monnig J, Kemp BE, Mattson MP (2001) AMP-activated protein kinase is highly expressed in neurons in the developing rat brain and promotes neuronal survival following glucose deprivation. J Mol Neurosci 17:45-58.

Cusack CL, Swahari V, Hampton Henley W, Michael Ramsey J, Deshmukh M (2013) Distinct pathways mediate axon degeneration during apoptosis and axon-specific pruning. Nat Commun 4:1876.

Dickey AS, Strack S (2011) PKA/AKAP1 and PP2A/Bbeta2 regulate neuronal morphogenesis via Drp1 phosphorylation and mitochondrial bioenergetics. J Neurosci 31:15716-15726.

Egan DF, Shackelford DB, Mihaylova MM, Gelino S, Kohnz RA, Mair W, Vasquez DS, Joshi A, Gwinn DM, Taylor R, Asara JM, Fitzpatrick J, Dillin A, Viollet B, Kundu M, Hansen M, Shaw RJ (2011) Phosphorylation of ULK1 (hATG1) by AMP-activated protein kinase connects energy sensing to mitophagy. Science 331:456-461.

Einheber S, Bhat MA, Salzer JL (2006) Disrupted axo-glial junctions result in accumulation of abnormal mitochondria at nodes of ranvier. Neuron glia Biol 2:165-174.

Glater EE, Megeath LJ, Stowers RS, Schwarz TL (2006) Axonal transport of mitochondria requires milton to recruit kinesin heavy chain and is light chain independent. J Cell Biol 173:545-557.

Hardie DG, Ross FA, Hawley SA (2012) AMPK: a nutrient and energy sensor that maintains energy homeostasis. Nat Rev Mol Cell Biol 13:251-262.
Herzig S, Shaw RJ (2018) AMPK: guardian of metabolism and mitochondrial homeostasis. Nat Rev Mol Cell Biol 19:121-135.

Izumi Y, Katsuki H, Zorumski CF (1997) Monocarboxylates (pyruvate and lactate) as alternative energy substrates for the induction of long-term potentiation in rat hippocampal slices. Neurosci Lett 232:17-20.

Kaasik A, Safiulina D, Choubey V, Kuum M, Zharkovsky A, Veksler V (2007) Mitochondrial swelling impairs the transport of organelles in cerebellar granule neurons. J Biol Chem 282:32821-32826.

Kang JS, Tian JH, Pan PY, Zald P, Li C, Deng C, Sheng ZH (2008) Docking of axonal mitochondria by syntaphilin controls their mobility and affects short-term facilitation. Cell 132:137-148.

Kann O, Kovács R (2007) Mitochondria and neuronal activity. Am J Physiol Cell Physiol 292:C641-C657.

Kaur C, Sivakumar V, Ang LS, Sundaresan A (2006) Hypoxic damage to the periventricular white matter in neonatal brain: role of vascular endothelial growth factor, nitric oxide and excitotoxicity. J Neurochem 98:12001216.

Kuznetsov SA, Langford GM, Weiss DG (1992) Actin-dependent organelle movement in squid axoplasm. Nature 356:722-725.

Li J, Zeng Z, Viollet B, Ronnett GV, McCullough LD (2007) Neuroprotective effects of adenosine monophosphate-activated protein kinase inhibition and gene deletion in stroke. Stroke; a journal of cerebral circulation 38:2992-2999.

Ligon LA, Steward O (2000) Movement of mitochondria in the axons and dendrites of cultured hippocampal neurons. J Comp Neur 427:340-350.

Llorens-Martín M, López-Doménech G, Soriano E, Avila J (2011) GSK3beta is involved in the relief of mitochondria pausing in a Tau-dependent manner. PLoS One 6:e27686.

Luders E, Steinmetz H, Jancke L (2002) Brain size and grey matter volume in the healthy human brain. Neuroreport 13:2371-2374

Magistretti PJ, Allaman I (2018) Lactate in the brain: from metabolic endproduct to signalling molecule. Nat Rev Neurosci 19:235-249.

Mattson MP, Partin J (1999) Evidence for mitochondrial control of neuronal polarity. J Neurosci Res 56:8-20.

McDonald A, Fogarty S, Leclerc I, Hill EV, Hardie DG, Rutter GA (2009) Control of insulin granule dynamics by AMPK dependent KLC1 phosphorylation. Islets 1:198-209.

Medana IM, Esiri MM (2003) Axonal damage: a key predictor of outcome in human CNS diseases. Brain 126:515-530.

Morris RL, Hollenbeck PJ (1993) The regulation of bidirectional mitochondrial transport is coordinated with axonal outgrowth. J of cell science 104: 917-927.

Morris RL, Hollenbeck PJ (1995) Axonal transport of mitochondria along microtubules and F-actin in living vertebrate neurons. J Cell Biol 131:1315-1326.

Neto E, Leitao L, Sousa DM, Alves CJ, Alencastre IS, Aguiar P, Lamghari M (2016) Compartmentalized Microfluidic Platforms: the Unrivaled Breakthrough of In Vitro Tools for Neurobiological Research. J Neurosci 36:11573-11584.

Pekkurnaz G, Trinidad JC, Wang X, Kong D, Schwarz TL (2014) Glucose regulates mitochondrial motility via Milton modification by O-GlcNAc transferase. Cell 158:54-68.

Pellerin L, Pellegri G, Bittar PG, Charnay Y, Bouras C, Martin JL, Stella N, Magistretti PJ (1998) Evidence supporting the existence of an activity-dependent astrocyte-neuron lactate shuttle. Dev Neurosci 20:291-299.

Pilling AD, Horiuchi D, Lively CM, Saxton WM (2006) Kinesin-1 and Dynein are the primary motors for fast transport of mitochondria in Drosophila motor axons. Mol Biol Cell 17:2057-2068.

Quintanilla RA, Dolan PJ, Jin YN, Johnson GV (2012) Truncated tau and Abeta cooperatively impair mitochondria in primary neurons. Neurobiology of aging 33:e625635.

Reck-Peterson SL, Yildiz A, Carter AP, Gennerich A, Zhang N, Vale RD (2006) Single-molecule analysis of dynein processivity and stepping behavior. Cell 126:335-348.

Reddy PH (2011) Abnormal tau, mitochondrial dysfunction, impaired axonal transport of mitochondria, and synaptic deprivation in Alzheimer's disease. Brain Res 1415:136-148.

Rintoul GL, Filiano AJ, Brocard JB, Kress GJ, Reynolds IJ (2003) Glutamate decreases mitochondrial size and movement in primary forebrain neurons. J Neurosci 23:7881-7888.

Sajic M, Mastrolia V, Lee CY, Trigo D, Sadeghian M, Mosley AJ, Gregson NA, Duchen MR, Smith KJ (2013) Impulse conduction increases 
mitochondrial transport in adult mammalian peripheral nerves in vivo. PLoS Biol 11:e1001754.

Schaffer BE, Levin RS, Hertz NT, Maures TJ, Schoof ML, Hollstein PE, Benayoun BA, Banko MR, Shaw RJ, Shokat KM, Brunet A (2015) Identification of AMPK Phosphorylation Sites Reveals a Network of Proteins Involved in Cell Invasion and Facilitates Large-Scale Substrate Prediction. Cell Metab 22:907-921.

Schnapp BJ, Reese TS (1989) Dynein is the motor for retrograde axonal transport of organelles. Proc Natl Acad Sci USA 86:1548-1552.

Schroer TA, Schnapp BJ, Reese TS, Sheetz MP (1988) The role of kinesin and other soluble factors in organelle movement along microtubules. J Cell Biol 107:1785-1792.

Tao K, Matsuki N, Koyama R (2014) AMP-activated protein kinase mediates activity-dependent axon branching by recruiting mitochondria to axon. Dev Neurobiol 74:557-573.

Taylor AM, Rhee SW, Tu CH, Cribbs DH, Cotman CW, Jeon NL (2003) Microfluidic Multicompartment Device for Neuroscience Research. Langmuir 19:1551-1556.

Taylor AM, Blurton-Jones M, Rhee SW, Cribbs DH, Cotman CW, Jeon NL (2005) A microfluidic culture platform for CNS axonal injury, regeneration and transport. Nat Methods 2:599-605.

Thornton C, Bright NJ, Sastre M, Muckett PJ, Carling D (2011) AMP-activated protein kinase (AMPK) is a tau kinase, activated in response to amyloid beta-peptide exposure. Biochem J 434:503-512.

Tuerk RD, Thali RF, Auchli Y, Rechsteiner H, Brunisholz RA, Schlattner U, Wallimann T, Neumann D (2007) New candidate targets of AMP- activated protein kinase in murine brain revealed by a novel multidimensional substrate-screen for protein kinases. J Proteome Res 6:3266-3277.

Turnley AM, Stapleton D, Mann RJ, Witters LA, Kemp BE, Bartlett PF (1999) Cellular distribution and developmental expression of AMP-activated protein kinase isoforms in mouse central nervous system. J Neurochem 72:1707-1716

Uchida A, Alami NH, Brown A (2009) Tight functional coupling of kinesin$1 \mathrm{~A}$ and dynein motors in the bidirectional transport of neurofilaments. Mol Biol Cell 20:4997-5006.

Weisova P, Concannon CG, Devocelle M, Prehn JH, Ward MW (2009) Regulation of glucose transporter 3 surface expression by the AMP-activated protein kinase mediates tolerance to glutamate excitation in neurons. J Neurosci 29:2997-3008.

Wender R, Brown AM, Fern R, Swanson RA, Farrell K, Ransom BR (2000) Astrocytic glycogen influences axon function and survival during glucose deprivation in central white matter. J Neurosci 20:6804-6810.

Zala D, Hinckelmann MV, Yu H, Lyra da Cunha MM, Liot G, Cordelieres FP, Marco S, Saudou F (2013) Vesicular glycolysis provides on-board energy for fast axonal transport. Cell 152:479-491.

Zheng Y, Zhang X, Wu X, Jiang L, Ahsan A, Ma S, Xiao Z, Han F, Qin ZH, $\mathrm{Hu}$ W, Chen Z (2019) Somatic autophagy of axonal mitochondria in ischemic neurons. J Cell Biol 218:1891-1907.

Zorova LD, Popkov VA, Plotnikov EY, Silachev DN, Pevzner IB, Jankauskas SS, Babenko VA, Zorov SD, Balakireva AV, Juhaszova M, Sollott SJ, Zorov DB (2018) Mitochondrial membrane potential. Anal Biochem 552:50-59. 